\title{
INTERVAL-VALUED INTUITIONISTIC FUZZY ORDERED PRECISE WEIGHTED AGGREGATION OPERATOR AND ITS APPLICATION IN GROUP DECISION MAKING
}

\author{
Wei ZHOU ${ }^{\mathrm{a}}$, Jian Min $\mathrm{HE}^{\mathrm{b}}$ \\ anternational Business School, Yunnan University of Finance and Economics, 650221 Kunming, \\ People's Republic of China \\ ${ }^{\mathrm{b} S}$ School of Economics and Management, Southeast University, 211189 Nanjing, People's Republic of China
}

Received 21 December 2011; accepted 19 May 2012

\begin{abstract}
An important research topic related to the theory and application of the interval-valued intuitionistic fuzzy weighted aggregation operators is how to determine their associated weights. In this paper, we propose a precise weight-determined (PWD) method of the monotonicity and scale-invariance, just based on the new score and accuracy functions of interval-valued intuitionistic fuzzy number (IIFN). Since the monotonicity and scale-invariance, the PWD method may be a precise and objective approach to calculate the weights of IIFN and interval-valued intuitionistic fuzzy aggregation operator, and a more suitable approach to distinguish different decision makers (DMs) and experts in group decision making. Based on the PWD method, we develop two new interval-valued intuitionistic fuzzy aggregation operators, i.e. interval-valued intuitionistic fuzzy ordered precise weighted averaging (IIFOPWA) operator and interval-valued intuitionistic fuzzy ordered precise weighted geometric (IIFOPWG) operator, and study their desirable properties in detail. Finally, we provide an illustrative example.
\end{abstract}

Keywords: multi-attribute group decision making, interval-valued intuitionistic fuzzy number, precise weight-determined method, monotonicity, scale-invariance.

JEL Classification: C02, D71, D81.

\section{Introduction}

Atanassov (1986) introduced the concept of intuitionistic fuzzy set (IFS), which is an extended and generalized definition of fuzzy set (FS) by Zadeh (1965), and is a powerful tool to deal with vagueness and uncertainty. Since its appearance, IFS has received more and more attention and applied to many fields (Vlachos, Sergiadis 2007; Xu, Yager 2008; Khatibi, Gholam 2009;

Corresponding author Wei Zhou

E-mail: zw453@163.com 
Kharal 2009; Ye 2010; Xu 2011; Zavadskas, Turskis 2011). However, decision making often occurs under the situation where there are time pressure and lack of data, and experts may not be able to express their preference between decisions with precise reasons. By combining FS and IFS, Atanassov and Gargov (1989) further proposed the concept of interval-valued intuitionistic fuzzy number (IIFN), which is characterized by a membership function and a non-membership function whose values are intervals rather than real numbers. Thus, the interval-valued intuitionistic fuzzy set has the virtue of complementing FS and IFS, which is more flexible and practical then FS and IFS in coping with fuzziness and uncertainty. After that, the interval-valued intuitionistic fuzzy set (IIFS) has received more and more attention from researchers (Deschrijver, Kerre 2003; Xu 2007a; John et al. 2009; Xu, Yager 2009; Li 2010a; Cabrerizoa et al. 2010; Ertugrul 2011), and obtained some research results (Park et al. 2009; Liu 2009; Li 2010b; Chen et al. 2011; Yue 2011; Zhou, He 2012).

For convenient aggregation and calculation the interval-valued intuitionistic fuzzy set, based on the weighted means and the ordered weighted averaging (OWA) functions (Yager 1988), Xu (2007b) investigated and proposed some interval-valued intuitionistic fuzzy aggregation operators, such as the interval-valued intuitionistic fuzzy weighted arithmetic aggregation (IIFWA) operator, the interval- valued intuitionistic fuzzy ordered weighted aggregation (IIFOWA) operator, and the interval-valued intuitionistic fuzzy hybrid aggregation (IIFHA) operator. Based on the GOWA operator (Yager 2004a) and intuitionistic fuzzy geometric aggregation operator (Xu, Yager 2006), Xu and Chen (2007a) proposed the interval-valued intuitionistic fuzzy ordered weighted geometric (IIFOWG) operator and the interval-valued intuitionistic fuzzy hybrid geometric (IIFHG) operator. Their analytical properties were explored by Wei and Wang (2007), and Xu and Chen (2007b). Moreover, Xu and Cai (2009) studied the incomplete interval-valued intuitionistic fuzzy preference relations and given an approach to decision making based on the incomplete interval-valued intuitionistic fuzzy preference relation. $\mathrm{Xu}$ (2010b) investigated the Choquet integrals of weighted intuitionistic fuzzy information. Base on the distance measure for interval-valued intuitionistic fuzzy value, Xu (2010c) further proposed some relations and operations of interval-valued intuitionistic fuzzy values. $\mathrm{Xu}$ and Chen (2011) proposed a Bonferroni means based on interval-valued intuitionistic fuzz value, and so on.

An important issue related to the theory and application of the above interval-valued intuitionistic fuzzy aggregation operators and other weighted aggregation operator is how to determine their weights (Yager 2004b). O’Hagan (1988) suggested a maximum entropy approach, which is called maximum entropy weights. Their properties were investigated by Filev and Yager (1995). Filev and Yager (1998) brought forward an exponential smoothing weighted method, which produces the so-called exponential OWA operator and operator weights. Other approaches include the genetic algorithm weighted method (Nettleton, Torra 2001), the minimum variance weighted method (Fuller, Majlender 2003), the parametric geometric weighted method (Liu, Chen 2004), the mini-max disparity weighted method (Wang, Parkan 2005), the maximal Renyi entropy weighted method (Majlender 2005), the preemptive goal programming weighted method (Wang, Parkan 2007), the deviation entropy weight method (Han, Liu 2011), and the distance measure weighted method (Yue 2011). 
However, there are still two puzzles to obtain interval-valued intuitionistic fuzzy aggregation operator weights by the above weight-determined methods. First, the weighted object of these approaches is the intuitionistic fuzzy value or the fuzzy value which is different from the interval-valued intuitionistic fuzzy number. Second, it seems that there is no investigation on the monotonous and scale-invariant properties of the weight-determined method, which is decisive for aggregation and calculation of interval-valued intuitionistic fuzzy values in group decision making. Therefore, how to calculate weights of the monotonicity and scale-invariance under the interval-valued intuitionistic fuzzy information environment is an important research topic that needs to be addressed in the process of multi-attribute group decision making, which is also the focus of this paper. To do this, we propose a precise weight-determined (PWD) method to obtain interval-valued intuitionistic fuzzy aggregation operator weights, based on the new score and accuracy functions of IIFN. Since the monotonicity and scale-invariance, the PWD method may be a precise and objective approach to calculate the weights of IIFN and interval-valued intuitionistic fuzzy aggregation operator, and a more suitable approach to distinguish different DMs and experts in group decision making. Then, based on the precise weight-determined method, we develop the interval-valued intuitionistic fuzzy ordered precise weighted averaging (IIFOPWA) operator and geometric (IIFOPWG) operator, and explore their desired properties in detail. At last, a practical example is provided to demonstrate the application of the new weighted method and two aggregation operators.

The reminder of this paper is organized as follows. We briefly review some basic concepts and operations in Section 1. Section 2 proposes the precise weight-determined method. Section 3 develops the IIFOPWA and IIFOPWG operators, and investigates their desired properties. A practical example is provided in Section 4 . The paper ends in final section with concluding remarks.

\section{Preliminaries}

In the following, we introduce the basic concepts and operations about IIFN (Xu 2007b), which is the basic element of IIFS (Atanassov, Gargov 1989).

Definition 1 (Atanassov, Gargov 1989). Let $X=\left(x_{1}, x_{2}, \cdots, x_{n}\right)$ be fixed. An interval-valued intuitionistic fuzzy set (IIFS) $\bar{A}$ in $X$ can be defined as:

$$
\bar{A}=\left\{\left(x, \bar{\mu}_{\bar{A}}(x), \bar{v}_{\bar{A}}(x)\right) \mid x \in X\right\},
$$

where $\bar{\mu}_{\bar{A}}(x) \subset[0,1]$ and $\bar{v}_{\bar{A}}(x) \subset[0,1]$ satisfy $\sup \bar{\mu}_{\bar{A}}(x)+\sup \bar{v}_{\bar{A}}(x) \leq 1$.

Definition 2 (Xu 2007b). Let $\bar{A}=\left\{\left(x, \bar{\mu}_{\bar{A}}(x), \bar{v}_{\bar{A}}(x)\right) \mid x \in X\right\}$ be an IIFS, the pair $\left(\bar{\mu}_{\bar{A}}(x), \bar{v}_{\bar{A}}(x)\right)$ is called an interval-valued intuitionistic fuzzy number (IIFN).

For computational convenience, an IIFN can be denoted by $([a, b],[c, d])$, with the condition that $[a, b] \subset[0,1],[c, d] \subset[0,1]$, and $b+d \leq 1$. Based on the operations of intuitionistic fuzzy values (Atanasov 1986; De et al. 2000), Xu (2007b) defined some operations of IIFNs as follows. 
Definition 3 (Xu 2007b). Let $\bar{\alpha}=([a, b],[c, d]), \bar{\alpha}_{1}=\left(\left[a_{1}, b_{1}\right],\left[c_{1}, d_{1}\right]\right)$ and $\bar{\alpha}_{2}=\left(\left[a_{2}, b_{2}\right],\left[c_{2}, d_{2}\right]\right)$ be three IIFNs, then following operational laws are valid:

1) $\bar{\alpha}_{1} \oplus \bar{\alpha}_{2}=\left(\left[a_{1}+a_{2}-a_{1} a_{2}, b_{1}+b_{2}-b_{1} b_{2}\right],\left[c_{1} c_{2}, d_{1} d_{2}\right]\right)$;

2) $\bar{\alpha}_{1} \otimes \bar{\alpha}_{2}=\left(\left[a_{1} a_{2}, b_{1} b_{2}\right],\left[c_{1}+c_{2}-c_{1} c_{2}, d_{1}+d_{2}-d_{1} d_{2}\right]\right)$;

3) $\lambda \bar{\alpha}=\left(\left[1-(1-a)^{\lambda}, 1-(1-b)^{\lambda},\right],\left[c^{\lambda}, d^{\lambda}\right]\right), \lambda>0$;

4) $\bar{\alpha}^{\lambda}=\left(\left[a^{\lambda}, b^{\lambda}\right],\left[1-(1-c)^{\lambda}, 1-(1-d)^{\lambda}\right]\right), \lambda>0$.

Then, $\mathrm{Xu}(2007 \mathrm{~b})$ introduced the score function $s(\bar{\alpha})=(a-c+b-d) / 2$ and the accuracy function $h(\bar{\alpha})=(a+b+c+d) / 2$ to calculate the score value and accuracy degree of IIFN $\bar{\alpha}=([a, b],[c, d])$, and gave an order relation between two IIFNs $\bar{\alpha}_{1}$ and $\bar{\alpha}_{2}$ as follows.

If $s\left(\bar{\alpha}_{1}\right)<s\left(\bar{\alpha}_{2}\right)$, then $\bar{\alpha}_{1}<\bar{\alpha}_{2}$.

If $s\left(\bar{\alpha}_{1}\right)=s\left(\bar{\alpha}_{2}\right)$, then:

i) if $h\left(\bar{\alpha}_{1}\right)<h\left(\bar{\alpha}_{2}\right)$, then $\bar{\alpha}_{1}<\bar{\alpha}_{2}$;

ii) if $h\left(\bar{\alpha}_{1}\right)=h\left(\bar{\alpha}_{2}\right)$, then $\bar{\alpha}_{1} \sim \bar{\alpha}_{2}$;

iii) if $h\left(\bar{\alpha}_{1}\right)>h\left(\bar{\alpha}_{2}\right)$, then $\bar{\alpha}_{1}>\bar{\alpha}_{2}$.

It should be noted that the score function $s(\bar{\alpha})$ is between -1 and 1 , and the accuracy function $h(\bar{\alpha})$ is between 0 and 2 . In order to facilitate the following study about operator weights, we respectively introduce new score function and accuracy function as follows:

$$
\begin{gathered}
s_{2}(\bar{\alpha})=(2+(a-c+b-d)) / 4 ; \\
h_{2}(\bar{\alpha})=(a+c+b+d) / 4 .
\end{gathered}
$$

Since $[a, b] \subset[0,1],[c, d] \subset[0,1]$, and $b+d \leq 1$, we can easily get $0 \leq s_{2}(\bar{\alpha}) \leq 1$ and $0 \leq h_{2}(\bar{\alpha}) \leq 1$. Compare $s(\bar{\alpha})$ and $s_{2}(\bar{\alpha})$, we can drive, if $s\left(\bar{\alpha}_{1}\right)>s\left(\bar{\alpha}_{2}\right)\left(\right.$ or $\left.s\left(\bar{\alpha}_{1}\right) \leq s\left(\bar{\alpha}_{2}\right)\right)$, then $s_{2}\left(\bar{\alpha}_{1}\right)>s_{2}\left(\bar{\alpha}_{2}\right)$ (or $s_{2}\left(\bar{\alpha}_{1}\right) \leq s_{2}\left(\bar{\alpha}_{2}\right)$ ); on the other hand, if $s_{2}\left(\bar{\alpha}_{1}\right)>s_{2}\left(\bar{\alpha}_{2}\right)$ (or $\left.s_{2}\left(\bar{\alpha}_{1}\right) \leq s_{2}\left(\bar{\alpha}_{2}\right)\right)$, then $s\left(\bar{\alpha}_{1}\right)>s\left(\bar{\alpha}_{2}\right)$ (or $\left.s\left(\bar{\alpha}_{1}\right) \leq s\left(\bar{\alpha}_{2}\right)\right)$. Likewise, we can get the same conclusions by comparing $h(\bar{\alpha})$ and $h_{2}(\bar{\alpha})$. Therefore, if we replace $s(\bar{\alpha})$ and $h(\bar{\alpha})$ by $s_{2}(\bar{\alpha})$ and $h_{2}(\bar{\alpha})$, the order relation between two IIFNs $\bar{\alpha}_{1}$ and $\bar{\alpha}_{2}$ introduced by Xu (2007b) are also valid.

Based on the above concepts and operations, $\mathrm{Xu}$ (2007b) proposed the following interval-valued intuitionistic fuzzy ordered weighted averaging (IIFOWA) operator and interval-valued intuitionistic fuzzy ordered weighted geometric (IIFOWG) operator to aggregate interval-valued intuitionistic fuzzy information.

Definition 4 (Xu 2007b). Let $\bar{\alpha}_{i}=\left(\left[a_{i}, b_{i}\right],\left[c_{i}, d_{i}\right]\right) \quad(i=1,2, \cdots, n)$ be a collection of IIFNs, with the condition that $\left[a_{i}, b_{i}\right] \subset[0,1],\left[c_{i}, d_{i}\right] \subset[0,1]$ and $b_{i}+d_{i} \leq 1$, IIFOWA operator and IIFOWG operator are defined as:

$$
\begin{aligned}
& \operatorname{IIFOWA}\left(\bar{\alpha}_{1}, \bar{\alpha}_{2}, \cdots, \bar{\alpha}_{n}\right)=w_{1} \bar{\alpha}_{\sigma(1)} \oplus w_{2} \bar{\alpha}_{\sigma(2)} \oplus \cdots \oplus w_{n} \bar{\alpha}_{\sigma(n)}= \\
& \left(\left[1-\prod_{i=1}^{n}\left(1-a_{\sigma(i)}\right)^{w_{i}}, 1-\prod_{i=1}^{n}\left(1-b_{\sigma(i)}\right)^{w_{i}}\right],\left[\prod_{i=1}^{n} c_{\sigma(i)} w_{i}, \prod_{i=1}^{n} d_{\sigma(i)} w_{i}\right]\right) ; \\
& \operatorname{IIFOWG}\left(\bar{\alpha}_{1}, \bar{\alpha}_{2}, \cdots, \bar{\alpha}_{n}\right)=\bar{\alpha}_{\sigma(1)}^{w_{1}} \otimes \bar{\alpha}_{\sigma(2)}^{w_{2}} \otimes \cdots \otimes \bar{\alpha}_{\sigma(n)}^{w_{n}}= \\
& \left(\left[\prod_{i=1}^{n} a_{\sigma(i)} w_{i}, \prod_{i=1}^{n} b_{\sigma(i)} w_{i}\right],\left[1-\prod_{i=1}^{n}\left(1-c_{\sigma(i)}\right)^{w_{i}}, 1-\prod_{i=1}^{n}\left(1-d_{\sigma(i)}\right)^{w_{i}}\right]\right),
\end{aligned}
$$


where $w=\left(w_{1}, w_{2}, \cdots, w_{n}\right)$ is the weight vector of $\bar{\alpha}_{i}$, with $w_{i} \in[0,1]$ and $\sum_{i=1}^{n} w_{i}=1$, and $\left(\bar{\alpha}_{\sigma(1)}, \bar{\alpha}_{\sigma(2)}, \cdots, \bar{\alpha}_{\sigma(n)}\right)$ is a permutation of $\left(\bar{\alpha}_{1}, \bar{\alpha}_{2}, \cdots, \bar{\alpha}_{n}\right)$ such that $\bar{\alpha}_{\sigma(i-1)} \geq \bar{\alpha}_{\sigma(i)}$ for $i=2,3, \cdots, n$.

\section{The precise weight-determined method under IIFN environment}

In this section, we shall propose a precise weight-determined method (PWD) for calculating interval-valued intuitionistic fuzzy aggregation operator weights. A prominent characteristic of PWD method is that put accuracy and precise weights into different IIFNs according to their score value and accuracy degree, and keep weights of the monotonicity and scale-invariance.

Definition 5. Let $\bar{\alpha}_{i}=\left(\left[a_{i}, b_{i}\right],\left[c_{i}, d_{i}\right]\right) \quad(i=1,2, \cdots, n)$ be a collection of IIFNs, and $\left(\bar{\alpha}_{\sigma(1)}, \bar{\alpha}_{\sigma(2)}, \cdots, \bar{\alpha}_{\sigma(n)}\right)$ is a permutation of $\left(\bar{\alpha}_{1}, \bar{\alpha}_{2}, \cdots, \bar{\alpha}_{n}\right)$ such that $\bar{\alpha}_{\sigma(i-1)} \geq \bar{\alpha}_{\sigma(i)}$, a precise weight vector $w=\left(w_{\sigma(1)}, w_{\sigma(2)}, \cdots, w_{\sigma(n)}\right)$ of $\bar{\alpha}_{i}$, is defined as follows:

where

$$
w_{\sigma(i)}=\frac{P_{\sigma(i)}}{\sum_{i}^{n} P_{\sigma(i)}},
$$

$$
\begin{gathered}
P_{\sigma(i)}=S\left(\bar{\alpha}_{\sigma(i)}\right) \cdot H\left(\bar{\alpha}_{\sigma(i)}\right) \cdot L\left(\bar{\alpha}_{\sigma(i)}\right) \cdot R\left(\bar{\alpha}_{\sigma(i)}\right), i=1,2, \cdots, n ; \\
S\left(\bar{\alpha}_{\sigma(i)}\right)=s_{2}\left(\bar{\alpha}_{\sigma(i)}\right) ; \\
L\left(\bar{\alpha}_{\sigma(i)}\right)=\prod_{j=1}^{i-1} l\left(\bar{\alpha}_{\sigma(j)}\right), i=2,3, \cdots, n ; \\
R\left(\bar{\alpha}_{\sigma(i)}\right)=\prod_{j=i+1}^{n} r\left(\bar{\alpha}_{\sigma(j)}\right), i=1,2, \cdots, n-1 ; \\
H\left(\bar{\alpha}_{\sigma(j)}\right)=\left\{\begin{array}{l}
h_{2}\left(\bar{\alpha}_{\sigma(i)}\right), \text { if } \prod_{j=1, j \neq i}^{n}\left(s_{2}\left(\bar{\alpha}_{\sigma(i)}\right)-s_{2}\left(\bar{\alpha}_{\sigma(j)}\right)\right)=0 ; \\
1 \quad \text { else } \\
j=2,3, \cdots, n-1 \\
r\left(\bar{\alpha}_{\sigma(j)}\right)=\left\{\begin{array}{c}
h_{2}\left(\bar{\alpha}_{\sigma(j)}\right), \text { if } s_{2}\left(\bar{\alpha}_{\sigma(j-1)}\right)-s_{2}\left(\bar{\alpha}_{\sigma(j)}\right)=0 \text { and } s_{2}\left(\bar{\alpha}_{\sigma(j)}\right)-s_{2}\left(\bar{\alpha}_{\sigma(j+1)}\right)>0 \\
h_{2}\left(\bar{\alpha}_{\sigma(j)}\right), \text { if } s_{2}\left(\bar{\alpha}_{\sigma(j-1)}\right)-s_{2}\left(\bar{\alpha}_{\sigma(j)}\right)>0 \text { and } s_{2}\left(\bar{\alpha}_{\sigma(j)}\right)-s_{2}\left(\bar{\alpha}_{\sigma(j+1)}\right)=0 \\
j=2,3, \cdots, n-1
\end{array},\right.
\end{array}\right.
\end{gathered}
$$

with $l\left(\bar{\alpha}_{\sigma(1)}\right)=l\left(\bar{\alpha}_{\sigma(n)}\right)=r\left(\bar{\alpha}_{\sigma(1)}\right)=r\left(\bar{\alpha}_{\sigma(n)}\right)=1, L\left(\bar{\alpha}_{\sigma(1)}\right)=1, R\left(\bar{\alpha}_{\sigma(n)}\right)=R\left(\bar{\alpha}_{\sigma(n-1)}\right)$.

By Definition 5, we can obtain $0 \leq w_{\sigma(i)} \leq 1$ and $\sum_{i=1}^{n} w_{\sigma(i)}=1$.

The above equations (Eqs (6)-(13)) are named precise weight-determined (PWD) method of obtaining the interval-valued intuitionistic fuzzy aggregation operator weights. In the following, we discuss its two desired properties. 
Theorem 1. (Monotonicity) Let $\bar{\alpha}_{i}=\left(\left[a_{i}, b_{i}\right],\left[c_{i}, d_{i}\right]\right) \quad(i=1,2, \cdots, n)$ be a collection of IIFNs, $\left(\bar{\alpha}_{\sigma(1)}, \bar{\alpha}_{\sigma(2)}, \cdots, \bar{\alpha}_{\sigma(n)}\right)$ is a permutation of $\left(\bar{\alpha}_{1}, \bar{\alpha}_{2}, \cdots, \bar{\alpha}_{n}\right)$ such that $\bar{\alpha}_{\sigma(i-1)} \geq \bar{\alpha}_{\sigma(i)}$, $\left(w_{\sigma(1)}, w_{\sigma(2)}, \cdots, w_{\sigma(n)}\right)$ is precise weight vector of $\bar{\alpha}_{\sigma(i)}$. Let $\bar{\alpha}_{\sigma(t 1)}$ and $\bar{\alpha}_{\sigma(t 2)}(t 1<t 2)$ be any two IIFNs in $\bar{\alpha}_{\sigma(i)}$, then

(1) if $\bar{\alpha}_{\sigma(t 1)}>\bar{\alpha}_{\sigma(t 2)}$, then $w_{\sigma(t 1)}>w_{\sigma(t 2)}$; (2) if $\bar{\alpha}_{\sigma(t 1)} \sim \bar{\alpha}_{\sigma(t 2)}$, then $w_{\sigma(t 1)}=w_{\sigma(t 2)}$.

Proof. (1) If $\bar{\alpha}_{\sigma(t))}>\bar{\alpha}_{\sigma(t 2)}$, we can distinguish eight following cases:

$s_{2}\left(\bar{\alpha}_{\sigma(t 1)}\right)>s_{2}\left(\bar{\alpha}_{\sigma(t 2)}\right), H\left(\bar{\alpha}_{\sigma(t 1)}\right)=1$, and $H\left(\bar{\alpha}_{\sigma(t 2)}\right)=1$;

$s_{2}\left(\bar{\alpha}_{\sigma(t 1)}\right)>s_{2}\left(\bar{\alpha}_{\sigma(t 2)}\right), H\left(\bar{\alpha}_{\sigma(t 1)}\right)<1$, and $H\left(\bar{\alpha}_{\sigma(t 2)}\right)=1$;

$s_{2}\left(\bar{\alpha}_{\sigma(t 1)}\right)>s_{2}\left(\bar{\alpha}_{\sigma(t 2)}\right), H\left(\bar{\alpha}_{\sigma(t 1)}\right)=1$, and $H\left(\bar{\alpha}_{\sigma(t 2)}\right)<1$;

$s_{2}\left(\bar{\alpha}_{\sigma(t 1)}\right)>s_{2}\left(\bar{\alpha}_{\sigma(t 2)}\right), H\left(\bar{\alpha}_{\sigma(t 1)}\right)<1$, and $H\left(\bar{\alpha}_{\sigma(t 2)}\right)<1$;

$s_{2}\left(\bar{\alpha}_{\sigma(t 1)}\right)=s_{2}\left(\bar{\alpha}_{\sigma(t 2)}\right), h_{2}\left(\bar{\alpha}_{\sigma(t 1)}\right)>h_{2}\left(\bar{\alpha}_{\sigma(t 2)}\right), H\left(\bar{\alpha}_{\sigma(t 1)}\right)=1$, and $H\left(\bar{\alpha}_{\sigma(t 2)}\right)=1$;

$s_{2}\left(\bar{\alpha}_{\sigma(t 1)}\right)=s_{2}\left(\bar{\alpha}_{\sigma(t 2)}\right), h_{2}\left(\bar{\alpha}_{\sigma(t 1)}\right)>h_{2}\left(\bar{\alpha}_{\sigma(t 2)}\right), H\left(\bar{\alpha}_{\sigma(t 1)}\right)<1$, and $H\left(\bar{\alpha}_{\sigma(t 2)}\right)=1$;

$s_{2}\left(\bar{\alpha}_{\sigma(t 1)}\right)=s_{2}\left(\bar{\alpha}_{\sigma(t 2)}\right), h_{2}\left(\bar{\alpha}_{\sigma(t 1)}\right)>h_{2}\left(\bar{\alpha}_{\sigma(t 2)}\right), H\left(\bar{\alpha}_{\sigma(t 1)}\right)=1$, and $H\left(\bar{\alpha}_{\sigma(t 2)}\right)<1$;

$s_{2}\left(\bar{\alpha}_{\sigma(t 1)}\right)=s_{2}\left(\bar{\alpha}_{\sigma(t 2)}\right), h_{2}\left(\bar{\alpha}_{\sigma(t 1)}\right)>h_{2}\left(\bar{\alpha}_{\sigma(t 2)}\right), H\left(\bar{\alpha}_{\sigma(t 1)}\right)<1$, and $H\left(\bar{\alpha}_{\sigma(t 2)}\right)<1$.

Here we only prove two cases, and the others can be obtained similarly.

Case 1: $s_{2}\left(\bar{\alpha}_{\sigma(t 1)}\right)>s_{2}\left(\bar{\alpha}_{\sigma(t 2)}\right), H\left(\bar{\alpha}_{\sigma(t 1)}\right)=1$, and $H\left(\bar{\alpha}_{\sigma(t 2)}\right)=1$.

In this case, we have:

$$
\begin{gathered}
S\left(\bar{\alpha}_{\sigma(t 1-1)}\right)>S\left(\bar{\alpha}_{\sigma(t 1)}\right)>S\left(\bar{\alpha}_{\sigma(t 1+1)}\right) ; \\
S\left(\bar{\alpha}_{\sigma(t 2-1)}\right)>S\left(\bar{\alpha}_{\sigma(t 2)}\right)>S\left(\bar{\alpha}_{\sigma(t 2+1)}\right) ; \\
l\left(\bar{\alpha}_{\sigma(t 1)}\right)=l\left(\bar{\alpha}_{\sigma(t 2)}\right)=r\left(\bar{\alpha}_{\sigma(t 1)}\right)=r\left(\bar{\alpha}_{\sigma(t 2)}\right)=1
\end{gathered}
$$

and

$$
\begin{aligned}
& L\left(\bar{\alpha}_{\sigma(t 1)}\right) \cdot R\left(\bar{\alpha}_{\sigma(t 1)}\right)= \\
& \prod_{j=1}^{t 1-1} l\left(\bar{\alpha}_{\sigma(j)}\right) \cdot \prod_{j=t 1+1}^{n} r\left(\bar{\alpha}_{\sigma(j)}\right)=\prod_{j=1}^{t 1-1} l\left(\bar{\alpha}_{\sigma(j)}\right) \cdot \prod_{j=t 1+1}^{t 2} r\left(\bar{\alpha}_{\sigma(j)}\right) \cdot \prod_{j=t 2+1}^{n} r\left(\bar{\alpha}_{\sigma(j)}\right) ; \\
& L\left(\bar{\alpha}_{\sigma(t 2)}\right) \cdot R\left(\bar{\alpha}_{\sigma(t 2)}\right)= \\
& \prod_{j=1}^{t 2-1} l\left(\bar{\alpha}_{\sigma(j)}\right) \cdot \prod_{j=t 2+1}^{n} r\left(\bar{\alpha}_{\sigma(j)}\right)=\prod_{j=1}^{t 1-1} l\left(\bar{\alpha}_{\sigma(j)}\right) \cdot \prod_{j=t 1}^{t 2-1} l\left(\bar{\alpha}_{\sigma(j)}\right) \cdot \prod_{j=t 2+1}^{n} r\left(\bar{\alpha}_{\sigma(j)}\right) ;
\end{aligned}
$$

i) if $s_{2}\left(\bar{\alpha}_{\sigma(t 1)}\right)>s_{2}\left(\bar{\alpha}_{\sigma(t 1+1)}\right)>\cdots>s_{2}\left(\bar{\alpha}_{\sigma(t 2)}\right)$, then

$$
\begin{aligned}
\prod_{j=t 1+1}^{t 2} r\left(\bar{\alpha}_{\sigma(j)}\right) & =\prod_{j=t 1}^{t 2-1} l\left(\bar{\alpha}_{\sigma(j)}\right)=1 ; \\
R\left(\bar{\alpha}_{\sigma(t 1)}\right) \cdot L\left(\bar{\alpha}_{\sigma(t 1)}\right) & =R\left(\bar{\alpha}_{\sigma(t 2)}\right) \cdot L\left(\bar{\alpha}_{\sigma(t 2)}\right) .
\end{aligned}
$$

Hence, $P_{\sigma(t 1)}>P_{\sigma(t 2)}$ and $w_{\sigma(t 1)}=\frac{P_{\sigma(t 1)}}{\sum_{i}^{n} P_{\sigma(i)}}>\frac{P_{\sigma(t 2)}}{\sum_{i}^{n} P_{\sigma(i)}}=w_{\sigma(t 2)}$;

ii) if $s_{2}\left(\bar{\alpha}_{\sigma(t 1)}\right)>\cdots>s_{2}\left(\bar{\alpha}_{\sigma(t 1+p)}\right)=\cdots=s_{2}\left(\bar{\alpha}_{\sigma(t 1+q)}\right)>\cdots>s_{2}\left(\bar{\alpha}_{\sigma(t 2)}\right)$, then

$$
h_{2}\left(\bar{\alpha}_{\sigma(t 1+p)}\right) \geq h_{2}\left(\bar{\alpha}_{\sigma(t 1+p+1)}\right) \geq \cdots \geq h_{2}\left(\bar{\alpha}_{\sigma(t 1+q)}\right)
$$


and

$$
\begin{aligned}
& \prod_{j=t 1+1}^{t 2} r\left(\bar{\alpha}_{\sigma(j)}\right)=1_{a_{\sigma(t 1+1)}} \times \cdots \times 1_{a_{\sigma(t 1+p-1)}} \times h_{2}\left(\bar{\alpha}_{\sigma(t 1+p)}\right)_{a_{\sigma(t 1+p)}} \times \\
& 1_{a_{\sigma(t 1+p+1)}} \times \cdots \times 1_{a_{\sigma(t 2)}}=h_{2}\left(\bar{\alpha}_{\sigma(t 1+p)}\right) ; \\
& \prod_{j=t 1}^{t 2-1} l\left(\bar{\alpha}_{\sigma(j)}\right)=1_{a_{\sigma(t 1)}} \times \cdots \times 1_{a_{\sigma(t 1+q-1)}} \times h_{2}\left(\bar{\alpha}_{\sigma(t 1+q)}\right)_{a_{\sigma(t 1+q)}} \times \\
& 1_{a_{\sigma(t 1+q+1)}} \times \cdots \times 1_{a_{\sigma(t 2-1)}}=h_{2}\left(\bar{\alpha}_{\sigma(t 1+q)}\right) .
\end{aligned}
$$

Therefore, $\prod_{j=t 1+1}^{t 2} r\left(\bar{\alpha}_{\sigma(j)}\right) \geq \prod_{j=t 1}^{t 2-1} l\left(\bar{\alpha}_{\sigma(j)}\right), P_{\sigma(t 1)}>P_{\sigma(t 2)}$ and

$$
w_{\sigma(t 1)}=\frac{P_{\sigma(t 1)}}{\sum_{i}^{n} P_{\sigma(i)}}>\frac{P_{\sigma(t 2)}}{\sum_{i}^{n} P_{\sigma(i)}}=w_{\sigma(t 2)} .
$$

Similarly, we could prove the more general case, i.e.

$$
\begin{aligned}
& s_{2}\left(\bar{\alpha}_{\sigma(t 1)}\right)>\cdots>s_{2}\left(\bar{\alpha}_{\sigma(t 1+k 1)}\right)=\cdots=s_{2}\left(\bar{\alpha}_{\sigma(t 1+k 2)}\right)>\cdots>s_{2}\left(\bar{\alpha}_{\sigma(t 1+p)}\right)=\cdots= \\
& { }_{2}\left(\bar{\alpha}_{\sigma(t 1+q)}\right)>\cdots>s_{2}\left(\bar{\alpha}_{\sigma(t 1+m 1)}\right)=\cdots=s_{2}\left(\bar{\alpha}_{\sigma(t 1+m 2)}\right)>\cdots>s_{2}\left(\bar{\alpha}_{\sigma(t 2)}\right) .
\end{aligned}
$$

Case 2: $s_{2}\left(\bar{\alpha}_{\sigma(t 1)}\right)>s_{2}\left(\bar{\alpha}_{\sigma(t 2)}\right), H\left(\bar{\alpha}_{\sigma(t 1)}\right)<1$, and $H\left(\bar{\alpha}_{\sigma(t 2)}\right)=1$.

In this case, we have:

$$
\begin{gathered}
s_{2}\left(\bar{\alpha}_{\sigma(t 1-g 1)}\right)=\cdots=s_{2}\left(\bar{\alpha}_{\sigma(t 1)}\right)=\cdots=s_{2}\left(\bar{\alpha}_{\sigma(t 1+g 2)}\right) ; \\
h_{2}\left(\bar{\alpha}_{\sigma(t 1-g 1)}\right) \geq \cdots \geq h_{2}\left(\bar{\alpha}_{\sigma(t 1)}\right) \geq \cdots \geq h_{2}\left(\bar{\alpha}_{\sigma(t 1+g 2)}\right) ; \\
s_{2}\left(\bar{\alpha}_{\sigma(t 2-1)}\right)>s_{2}\left(\bar{\alpha}_{\sigma(t 2)}\right)>s_{2}\left(\bar{\alpha}_{\sigma(t 2+1)}\right),
\end{gathered}
$$

then

$$
\begin{aligned}
& H\left(\bar{\alpha}_{\sigma(t 1)}\right) L\left(\bar{\alpha}_{\sigma(t 1)}\right) R\left(\bar{\alpha}_{\sigma(t 1)}\right)=h_{2}\left(\bar{\alpha}_{\sigma(t 1)}\right) \cdot \prod_{j=1}^{t 1-1} l\left(\bar{\alpha}_{\sigma(j)}\right) \cdot \prod_{j=t 1+1}^{n} r\left(\bar{\alpha}_{\sigma(j)}\right)= \\
& h_{2}\left(\bar{\alpha}_{\sigma(t 1)}\right) \prod_{j=1}^{t 1-1} l\left(\bar{\alpha}_{\sigma(j)}\right) \cdot \prod_{j=t 1+1}^{t 2} r\left(\bar{\alpha}_{\sigma(j)}\right) \cdot \prod_{j=t 2+1}^{n} r\left(\bar{\alpha}_{\sigma(j)}\right) ; \\
& H\left(\bar{\alpha}_{\sigma(t 2)}\right) L\left(\bar{\alpha}_{\sigma(t 2)}\right) R\left(\bar{\alpha}_{\sigma(t 2)}\right)=\prod_{j=1}^{t 2-1} l\left(\bar{\alpha}_{\sigma(j)}\right) \cdot \prod_{j=t 2+1}^{n} r\left(\bar{\alpha}_{\sigma(j)}\right)= \\
& \prod_{j=1}^{t 1-1} l\left(\bar{\alpha}_{\sigma(j)}\right) \cdot \prod_{j=t 1}^{t 2-1} l\left(\bar{\alpha}_{\sigma(j)}\right) \cdot \prod_{j=t 2+1}^{n} r\left(\bar{\alpha}_{\sigma(j)}\right) ;
\end{aligned}
$$
have:

i) if $s_{2}\left(\bar{\alpha}_{\sigma(t 1-g 1)}\right)=s_{2}\left(\bar{\alpha}_{\sigma(t 1)}\right)=\cdots=s_{2}\left(\bar{\alpha}_{\sigma(t 1+g 2)}\right)>s_{2}\left(\bar{\alpha}_{\sigma(t 1+g 2+1)}\right)>\cdots>s_{2}\left(\bar{\alpha}_{\sigma(t 2)}\right)$, we

$$
\begin{gathered}
\prod_{j=t 1+1}^{t 2} r\left(\bar{\alpha}_{\sigma(j)}\right)=1 ; \\
\prod_{j=t 1}^{t 2-1} l\left(\bar{\alpha}_{\sigma(j)}\right)=h_{2}\left(\bar{\alpha}_{\sigma(t 1+g 2)}\right)_{\sigma(t 1+g 2)}=h_{2}\left(\bar{\alpha}_{\sigma(t 1+g 2)}\right) .
\end{gathered}
$$


Since $h_{2}\left(\bar{\alpha}_{\sigma(t 1)}\right) \geq h_{2}\left(\bar{\alpha}_{\sigma(t 1+g 2)}\right)$, then

$$
\begin{gathered}
H\left(\bar{\alpha}_{\sigma(t 1)}\right) \cdot L\left(\bar{\alpha}_{\sigma(t 1)}\right) \cdot R\left(\bar{\alpha}_{\sigma(t 1)}\right) \geq H\left(\bar{\alpha}_{\sigma(t 2)}\right) \cdot L\left(\bar{\alpha}_{\sigma(t 2)}\right) \cdot R\left(\bar{\alpha}_{\sigma(t 2)}\right) ; \\
w_{\sigma(t 1)}=\frac{P_{\sigma(t 1)}}{\sum_{i}^{n} P_{\sigma(i)}}>\frac{P_{\sigma(t 2)}}{\sum_{i}^{n} P_{\sigma(i)}}=w_{\sigma(t 2)} ;
\end{gathered}
$$

ii) if $s_{2}\left(\bar{\alpha}_{\sigma(t 1-g 1)}\right)=\cdots=s_{2}\left(\bar{\alpha}_{\sigma(t 1+g 2)}\right)>\cdots>s_{2}\left(\bar{\alpha}_{\sigma(t 1+p)}\right)=\cdots=s_{2}\left(\bar{\alpha}_{\sigma(t 1+q)}\right)>\cdots>s_{2}\left(\bar{\alpha}_{\sigma(t 2)}\right)$, we have:

$$
h_{2}\left(\alpha_{\sigma(t 1+p)}\right) \geq \ldots \geq\left(\alpha_{\sigma(t 1+q)}\right)
$$

and

$$
\begin{gathered}
\prod_{j=t 1+1}^{t 2} r\left(\bar{\alpha}_{\sigma(j)}\right)=1_{a_{\sigma(t 1+1)}} \times \cdots \times 1_{a_{\sigma(t 1+p-1)}} \times \\
h_{2}\left(\bar{\alpha}_{\sigma(t 1+p)}\right)_{a_{\sigma(t 1+p)}} \times 1_{a_{\sigma(t 1+p+1)}} \times \cdots \times 1_{a_{\sigma(t 2)}}=h_{2}\left(\bar{\alpha}_{\sigma(t 1+p)}\right) ; \\
\prod_{j=t 1}^{t 2-1} l\left(\bar{\alpha}_{\sigma(j)}\right)=1_{a_{\sigma(t 1)}} \times \cdots \times 1_{a_{\sigma(t 1+g 2-1)}} \times h_{2}\left(\bar{\alpha}_{\sigma(t 1+\mathrm{g} 2)}\right)_{a_{\sigma(t 1+g 2)}} \times 1_{a_{\sigma(t 1+g 2+1)}} \times \cdots \times \\
1_{a_{\sigma(t 1+q-1)}} \times h_{2}\left(\bar{\alpha}_{\sigma(t 1+q)}\right)_{a_{\sigma(t 1+q)}} \times 1_{a_{\sigma(t 1+q+1)}} \times \cdots \times 1_{a_{\sigma(t 2-1)}}=h_{2}\left(\bar{\alpha}_{\sigma(t 1+g 2)}\right) \cdot h_{2}\left(\bar{\alpha}_{\sigma(t 1+q)}\right) .
\end{gathered}
$$

Hence, $h_{2}\left(\alpha_{\sigma(t 1)}\right) \cdot \prod_{j=t 1+1}^{t 2} r\left(\alpha_{\sigma(j)}\right) \geq \prod_{j=t 1}^{t 2-1} l\left(\alpha_{\sigma(j)}\right), \quad P_{\sigma(t 1)}>P_{\sigma(t 2)}$, and

$$
w_{\sigma(t 1)}=\frac{P_{\sigma(t 1)}}{\sum_{i}^{n} P_{\sigma(i)}}>\frac{P_{\sigma(t 2)}}{\sum_{i}^{n} P_{\sigma(i)}}=w_{\sigma(t 2)} .
$$

Similarly, we could prove the more general case, i.e.

$$
\begin{aligned}
& s_{2}\left(\bar{\alpha}_{\sigma(t 1-g 1)}\right)=\cdots=s_{2}\left(\bar{\alpha}_{\sigma(t 1)}\right)=\cdots=s_{2}\left(\bar{\alpha}_{\sigma(t 1+g 2)}\right)>\cdots>s_{2}\left(\bar{\alpha}_{\sigma(t 1+k 1)}\right)= \\
& \cdots=s_{2}\left(\bar{\alpha}_{\sigma(t 1+k 2)}\right)>\cdots>s_{2}\left(\bar{\alpha}_{\sigma(t 1+p)}\right)=\cdots=s_{2}\left(\bar{\alpha}_{\sigma(t 1+q)}\right)>\cdots>s_{2}\left(\bar{\alpha}_{\sigma(t 1+m 1)}\right)= \\
& \cdots=s_{2}\left(\bar{\alpha}_{\sigma(t 1+m 2)}\right)>\cdots>s_{2}\left(\bar{\alpha}_{\sigma(t 2)}\right) ;
\end{aligned}
$$

(2) If $\bar{\alpha}_{\sigma(t 1)} \sim \bar{\alpha}_{\sigma(t 2)}$, then

$$
\begin{gathered}
\bar{\alpha}_{\sigma(t 1)} \sim \bar{\alpha}_{\sigma(t 1+1)} \sim \cdots \sim \bar{\alpha}_{\sigma(t 2)} ; \\
s_{2}\left(\bar{\alpha}_{\sigma(t 1)}\right)=s_{2}\left(\bar{\alpha}_{\sigma(t 1+1)}\right)=\cdots=s_{2}\left(\bar{\alpha}_{\sigma(t 2)}\right) ; \\
h_{2}\left(\bar{\alpha}_{\sigma(t 1)}\right)=h_{2}\left(\bar{\alpha}_{\sigma(t 1+1)}\right)=\cdots=h_{2}\left(\bar{\alpha}_{\sigma(t 2)}\right)
\end{gathered}
$$

and

$$
\begin{gathered}
S\left(\bar{\alpha}_{\sigma(t 1)}\right)=S\left(\bar{\alpha}_{\sigma(t 2)}\right) ; \\
H\left(\bar{\alpha}_{\sigma(t 1)}\right)=h_{2}\left(\bar{\alpha}_{\sigma(t 1)}\right)=H\left(\bar{\alpha}_{\sigma(t 2)}\right)=h_{2}\left(\bar{\alpha}_{\sigma(t 2)}\right) ;
\end{gathered}
$$

Since $\bar{\alpha}_{\sigma(t 1)}=\bar{\alpha}_{\sigma(t 1+1)}=\cdots=\bar{\alpha}_{\sigma(t 2)}$ and $s_{2}\left(\bar{\alpha}_{\sigma(t 1)}\right)=s_{2}\left(\bar{\alpha}_{\sigma(t 1+1)}\right)=\cdots=s_{2}\left(\bar{\alpha}_{\sigma(t 2)}\right)$, we have 


$$
\begin{gathered}
l\left(\bar{\alpha}_{\sigma(t 1)}\right)=l\left(\bar{\alpha}_{\sigma(t 1+1)}\right)=\cdots=l\left(\bar{\alpha}_{\sigma(t 2-1)}\right)=1 ; \\
r\left(\bar{\alpha}_{\sigma(t 1+1)}\right)=r\left(\bar{\alpha}_{\sigma(t 1+2)}\right)=\cdots=r\left(\bar{\alpha}_{\sigma(t 2)}\right)=1,
\end{gathered}
$$

then

$$
\begin{gathered}
L\left(\bar{\alpha}_{\sigma(t 2)}\right)=\prod_{j=1}^{t 2-1} l\left(\bar{\alpha}_{\sigma(j)}\right)=\prod_{j=1}^{t 1-1} l\left(\bar{\alpha}_{\sigma(j)}\right) \cdot \prod_{j=t 1}^{t 2-1} l\left(\bar{\alpha}_{\sigma(j)}\right)=L\left(\bar{\alpha}_{\sigma(t 1)}\right) ; \\
R\left(\bar{\alpha}_{\sigma(t 1)}\right)=\prod_{j=t 1+1}^{n} r\left(\bar{\alpha}_{\sigma(j)}\right)=\prod_{j=t 1+1}^{t 2} r\left(\bar{\alpha}_{\sigma(j)}\right) \cdot \prod_{j=t 2+1}^{n} r\left(\bar{\alpha}_{\sigma(j)}\right)=R\left(\bar{\alpha}_{\sigma(t 2)}\right) .
\end{gathered}
$$

Hence, $P_{\sigma(t 1)}=P_{\sigma(t 2)}$ and $w_{\sigma(t 1)}=\frac{P_{\sigma(t 1)}}{\sum_{i}^{n} P_{\sigma(i)}}=\frac{P_{\sigma(t 2)}}{\sum_{i}^{n} P_{\sigma(i)}}=w_{\sigma(t 2)}$.

Thus the proof is completed.

Theorem 1 indicates that the PWD method could put different weights into different IIFNs, just based on their score value and accuracy degree, which also demonstrates the monotonicity.

Theorem 2. (Scale-invariance) Assume that $\bar{\alpha}_{i}=\left(\left[a_{i}, b_{i}\right],\left[c_{i}, d_{i}\right]\right)(i=1,2, \cdots, n)$ is a collection of IIFNs, $\bar{\alpha}_{\sigma(i)}$ is a permutation of $\bar{\alpha}_{i}$ such that $\bar{\alpha}_{\sigma(i-1)} \geq \bar{\alpha}_{\sigma(i)}, \bar{\alpha}_{\sigma(t 1)}$ and $\bar{\alpha}_{\sigma(t 2)}$ $(t 1<t 2)$ are any two IIFNs in $\bar{\alpha}_{\sigma(i)}, w=\left(w_{\sigma(1)}, w_{\sigma(2)}, \cdots, w_{\sigma(n)}\right)$ is precise weight vector of IIFNs $\bar{\alpha}_{\sigma(i)}$, and $t i, t j \in\{t 1, t 1+1, \cdots, t 2\}$, then

(1) if $s_{2}\left(\bar{\alpha}_{\sigma(t 1)}\right)>s_{2}\left(\bar{\alpha}_{\sigma(t 1+1)}\right)>\cdots>s_{2}\left(\bar{\alpha}_{\sigma(t 2)}\right)$, then $\frac{w_{\sigma(t i)}}{w_{\sigma(t j)}}=\frac{s_{2}\left(\bar{\alpha}_{\sigma(t i)}\right)}{s_{2}\left(\bar{\alpha}_{\sigma(t j)}\right)}$;

(2) if $s_{2}\left(\bar{\alpha}_{\sigma(t 1)}\right)=s_{2}\left(\bar{\alpha}_{\sigma(t 1+1)}\right)=\cdots=s_{2}\left(\bar{\alpha}_{\sigma(t 2)}\right)$, then $\frac{w_{\sigma(t i)}}{w_{\sigma(t j)}}=\frac{h_{2}\left(\bar{\alpha}_{\sigma(t i)}\right)}{h_{2}\left(\bar{\alpha}_{\sigma(t j)}\right)}$.

Proof. For any IIFNs $\bar{\alpha}_{\sigma(t i)}$ and $\bar{\alpha}_{\sigma(t j)}$, we proof (1) and (2) as follows:

(1) If $s_{2}\left(\bar{\alpha}_{\sigma(t 1)}\right)>s_{2}\left(\bar{\alpha}_{\sigma(t 1+1)}\right)>\cdots>s_{2}\left(\bar{\alpha}_{\sigma(t 2)}\right), s_{2}\left(\bar{\alpha}_{\sigma(t 1-1)}\right)>s_{2}\left(\bar{\alpha}_{\sigma(t 1)}\right)$, and $s_{2}\left(\bar{\alpha}_{\sigma(t 2)}\right)>s_{2}\left(\bar{\alpha}_{\sigma(t 2+1)}\right)$, we have:

$$
\begin{gathered}
H\left(\bar{\alpha}_{\sigma(t 1)}\right)=H\left(\bar{\alpha}_{\sigma(t 1+1)}\right)=\cdots=H\left(\bar{\alpha}_{\sigma(t 2)}\right)=1 ; \\
L\left(\bar{\alpha}_{\sigma(t 1)}\right)=L\left(\bar{\alpha}_{\sigma(t 1+1)}\right)=\cdots=L\left(\bar{\alpha}_{\sigma(t 2)}\right)=\prod_{j=1}^{t 1-1} l\left(\bar{\alpha}_{\sigma(j)}\right) ; \\
R\left(\bar{\alpha}_{\sigma(t 1)}\right)=R\left(\bar{\alpha}_{\sigma(t 1+1)}\right)=\cdots=R\left(\bar{\alpha}_{\sigma(t 2)}\right)=\prod_{j=t 2+1}^{n} r\left(\bar{\alpha}_{\sigma(j)}\right),
\end{gathered}
$$

then

$$
\frac{w_{\sigma(t i)}}{w_{\sigma(t j)}}=\frac{P_{\sigma(t i)}}{P_{\sigma(t j)}}=\frac{s_{2}\left(\bar{\alpha}_{\sigma(t i)}\right)}{s_{2}\left(\bar{\alpha}_{\sigma(t j)}\right)}, i, j=t 1, t 1+1, \cdots, t 2 .
$$

If $s_{2}\left(\bar{\alpha}_{\sigma(t 1)}\right)>s_{2}\left(\bar{\alpha}_{\sigma(t 1+1)}\right)>\cdots>s_{2}\left(\bar{\alpha}_{\sigma(t 2)}\right), s_{2}\left(\bar{\alpha}_{\sigma(t 1-1)}\right)>s_{2}\left(\bar{\alpha}_{\sigma(t 1)}\right)$, $s_{2}\left(\bar{\alpha}_{\sigma(t 2)}\right)=s_{2}\left(\bar{\alpha}_{\sigma(t 2+1)}\right)$, we have:

$$
\begin{gathered}
H\left(\bar{\alpha}_{\sigma(t 1)}\right)=H\left(\bar{\alpha}_{\sigma(t 1+1)}\right)=\cdots=H\left(\bar{\alpha}_{\sigma(t 2-1)}\right)=1 ; \\
H\left(\bar{\alpha}_{\sigma(t 2)}\right)=h_{2}\left(\bar{\alpha}_{\sigma(t 2)}\right) ;
\end{gathered}
$$




$$
\begin{gathered}
L\left(\bar{\alpha}_{\sigma(t 1)}\right)=L\left(\bar{\alpha}_{\sigma(t 1+1)}\right)=\cdots=L\left(\bar{\alpha}_{\sigma(t 2)}\right)=\prod_{j=1}^{t 1-1} l\left(\bar{\alpha}_{\sigma(j)}\right) ; \\
R\left(\bar{\alpha}_{\sigma(t 1)}\right)=R\left(\bar{\alpha}_{\sigma(t 1+1)}\right)=\cdots=R\left(\bar{\alpha}_{\sigma(t 2-1)}\right)=h_{2}\left(\bar{\alpha}_{\sigma(t 2)}\right) \cdot \prod_{j=t 2+1}^{n} r\left(\bar{\alpha}_{\sigma(j)}\right) ; \\
R\left(\bar{\alpha}_{\sigma(t 2)}\right)=\prod_{j=t 2+1}^{n} r\left(\bar{\alpha}_{\sigma(j)}\right),
\end{gathered}
$$

then

$$
\frac{w_{\sigma(t i)}}{w_{\sigma(t j)}}=\frac{P_{\sigma(t i)}}{P_{\sigma(t j)}}=\frac{s_{2}\left(\bar{\alpha}_{\sigma(t i)}\right)}{s_{2}\left(\bar{\alpha}_{\sigma(t j)}\right)}, i, j=t 1, t 1+1, \cdots, t 2 .
$$

We could similarly prove other two cases, such as:

i) $s_{2}\left(\bar{\alpha}_{\sigma(t 1)}\right)>s_{2}\left(\bar{\alpha}_{\sigma(t 1+1)}\right)>\cdots>s_{2}\left(\bar{\alpha}_{\sigma(t 2)}\right), s_{2}\left(\bar{\alpha}_{\sigma(t 1-1)}\right)=s_{2}\left(\bar{\alpha}_{\sigma(t 1)}\right)$, and $s_{2}\left(\bar{\alpha}_{\sigma(t 1-1)}\right)>s_{2}\left(\bar{\alpha}_{\sigma(t 1)}\right)$;

ii) $s_{2}\left(\bar{\alpha}_{\sigma(t 1)}\right)>s_{2}\left(\bar{\alpha}_{\sigma(t 1+1)}\right)>\cdots>s_{2}\left(\bar{\alpha}_{\sigma(t 2)}\right), s_{2}\left(\bar{\alpha}_{\sigma(t 1-1)}\right)=s_{2}\left(\bar{\alpha}_{\sigma(t 1)}\right)$, and $s_{2}\left(\bar{\alpha}_{\sigma(t 1-1)}\right)=s_{2}\left(\bar{\alpha}_{\sigma(t 1)}\right)$.

(2) If $s_{2}\left(\bar{\alpha}_{\sigma(t 1)}\right)=s_{2}\left(\bar{\alpha}_{\sigma(t 1+1)}\right)=\cdots=s_{2}\left(\bar{\alpha}_{\sigma(t 2)}\right), s_{2}\left(\bar{\alpha}_{\sigma(t 1-1)}\right)>s_{2}\left(\bar{\alpha}_{\sigma(t 1)}\right)$, and $s_{2}\left(\bar{\alpha}_{\sigma(t 2)}\right)>s_{2}\left(\bar{\alpha}_{\sigma(t 2+1)}\right)$, we have:

$$
\begin{gathered}
H\left(\bar{\alpha}_{\sigma(i)}\right)=h_{2}\left(\bar{\alpha}_{\sigma(i)}\right), i=t 1, t 1+1, \cdots, t 2 ; \\
L\left(\bar{\alpha}_{\sigma(t 1)}\right)=L\left(\bar{\alpha}_{\sigma(t 1+1)}\right)=\cdots=L\left(\bar{\alpha}_{\sigma(t 2)}\right)=h_{2}\left(\bar{\alpha}_{\sigma(t 1)}\right) \cdot \prod_{j=1}^{t 1-1} l\left(\bar{\alpha}_{\sigma(j)}\right) ; \\
R\left(\bar{\alpha}_{\sigma(t 1)}\right)=R\left(\bar{\alpha}_{\sigma(t 1+1)}\right)=\cdots=R\left(\bar{\alpha}_{\sigma(t 2)}\right)=h_{2}\left(\bar{\alpha}_{\sigma(t 2)}\right) \cdot \prod_{j=t 2+1}^{n} r\left(\bar{\alpha}_{\sigma(j)}\right),
\end{gathered}
$$

then

$$
\frac{w_{\sigma(t i)}}{w_{\sigma(t j)}}=\frac{P_{\sigma(t i)}}{P_{\sigma(t j)}}=\frac{h_{2}\left(\bar{\alpha}_{\sigma(t i)}\right)}{h_{2}\left(\bar{\alpha}_{\sigma(t j)}\right)}, i, j=t 1, t 1+1, \cdots, t 2 . .
$$

We could similarly prove other three cases, i.e.

i) $s_{2}\left(\bar{\alpha}_{\sigma(t 1)}\right)=\cdots=s_{2}\left(\bar{\alpha}_{\sigma(t 2)}\right), s_{2}\left(\bar{\alpha}_{\sigma(t 1-1)}\right)>s_{2}\left(\bar{\alpha}_{\sigma(t 1)}\right), s_{2}\left(\bar{\alpha}_{\sigma(t 2)}\right)=s_{2}\left(\bar{\alpha}_{\sigma(t 2+1)}\right)$;

ii) $s_{2}\left(\bar{\alpha}_{\sigma(t 1)}\right)=\cdots=s_{2}\left(\bar{\alpha}_{\sigma(t 2)}\right), s_{2}\left(\bar{\alpha}_{\sigma(t 1-1)}\right)=s_{2}\left(\bar{\alpha}_{\sigma(t 1)}\right), s_{2}\left(\bar{\alpha}_{\sigma(t 2)}\right)=s_{2}\left(\bar{\alpha}_{\sigma(t 2+1)}\right)$;

iii) $s_{2}\left(\bar{\alpha}_{\sigma(t 1)}\right)=\cdots=s_{2}\left(\bar{\alpha}_{\sigma(t 2)}\right), s_{2}\left(\bar{\alpha}_{\sigma(t 1-1)}\right)=s_{2}\left(\bar{\alpha}_{\sigma(t 1)}\right), s_{2}\left(\bar{\alpha}_{\sigma(t 2)}\right)>s_{2}\left(\bar{\alpha}_{\sigma(t 2+1)}\right)$.

Thus the proof is completed.

Example 1. Let $\bar{\alpha}_{1}=([0.1,0.8],[0.1,0.2]), \bar{\alpha}_{2}=([0.1,0.7],[0.1,0.2]), \bar{\alpha}_{3}=([0.3,0.6],[0.2,0.3])$, $\bar{\alpha}_{4}=([0.2,0.6],[0.1,0.3])$ and $\bar{\alpha}_{5}=([0.2,0.5],[0.1,0.4])$ be five interval-valued intuitionistic fuzzy numbers (IIFNs) expressed by five DMs (decision makers). To aggregate these attribute values and reflect DMs' preference, we calculate DMs' weights used the prioritized weighted $(\mathrm{Xu}$, Sun 2011) and PWD methods respectively as follows.

According to Eqs (2) and (3), we have:

$$
s_{2}\left(\bar{\alpha}_{1}\right)=0.65, h_{2}\left(\bar{\alpha}_{1}\right)=0.30, s_{2}\left(\bar{\alpha}_{2}\right)=0.625, h_{2}\left(\bar{\alpha}_{2}\right)=0.275, s_{2}\left(\bar{\alpha}_{3}\right)=0.60 ;
$$




$$
h_{2}\left(\bar{\alpha}_{3}\right)=0.35, s_{2}\left(\bar{\alpha}_{4}\right)=0.60, h_{2}\left(\bar{\alpha}_{4}\right)=0.30, s_{2}\left(\bar{\alpha}_{5}\right)=0.55, h_{2}\left(\bar{\alpha}_{5}\right)=0.30,
$$

then $s_{2}\left(\bar{\alpha}_{1}\right)>s_{2}\left(\bar{\alpha}_{2}\right)>s_{2}\left(\bar{\alpha}_{3}\right)=s_{2}\left(\bar{\alpha}_{4}\right)>s_{2}\left(\bar{\alpha}_{5}\right)$ and $\bar{\alpha}_{1} \succ \bar{\alpha}_{2} \succ \bar{\alpha}_{3} \succ \bar{\alpha}_{4} \succ \bar{\alpha}_{5}$.

1. If we use the prioritized weighted method to calculate, then we have:

$$
\begin{gathered}
V_{0}=1, V_{1}=s_{2}\left(\bar{\alpha}_{1}\right)=0.65, V_{2}=s_{2}\left(\bar{\alpha}_{4}\right)=0.60 ; \\
V_{3}=s_{2}\left(\bar{\alpha}_{3}\right)=0.60, V_{4}=s_{2}\left(\bar{\alpha}_{4}\right)=0.60, V_{5}=s_{2}\left(\bar{\alpha}_{5}\right)=0.55 .
\end{gathered}
$$

Therefore,

$$
\begin{gathered}
\mathrm{T}_{1}=V_{0}=1, \mathrm{~T}_{2}=\prod_{k=1}^{1} V_{\sigma(k)}=V_{\sigma(1)}=0.65, \mathrm{~T}_{3}=\prod_{k=1}^{2} V_{\sigma(k)}=0.39 ; \\
\mathrm{T}_{4}=\prod_{k=1}^{3} V_{\sigma(k)}=0.234, \mathrm{~T}_{5}=\prod_{k=1}^{4} V_{\sigma(k)}=0.1278
\end{gathered}
$$

and

Thus,

$$
\begin{gathered}
\text { and } w_{1}\left(\bar{\alpha}_{\sigma(1)}\right)=\frac{\mathrm{T}_{1}}{\sum_{i=1}^{5} \mathrm{~T}_{i}}=0.4148, w_{1}\left(\bar{\alpha}_{\sigma(2)}\right)=\frac{\mathrm{T}_{2}}{\sum_{i=1}^{5} \mathrm{~T}_{i}}=0.2696, w_{1}\left(\bar{\alpha}_{\sigma(3)}\right)=\frac{\mathrm{T}_{3}}{\sum_{i=1}^{5} \mathrm{~T}_{i}}=0.1618 ; \\
w_{1}\left(\bar{\alpha}_{\sigma(4)}\right)=\frac{\mathrm{T}_{4}}{\sum_{i=1}^{5} \mathrm{~T}_{i}}=0.1008, w_{1}\left(\bar{\alpha}_{\sigma(5)}\right)=\frac{\mathrm{T}_{5}}{\sum_{i=1}^{5} \mathrm{~T}_{i}}=0.0530 .
\end{gathered}
$$

$$
\left(w_{1}\left(\bar{\alpha}_{\sigma(1)}\right), w_{1}\left(\bar{\alpha}_{\sigma(2)}\right), w_{1}\left(\bar{\alpha}_{\sigma(3)}\right), w_{1}\left(\bar{\alpha}_{\sigma(4)}\right), w_{1}\left(\bar{\alpha}_{\sigma(5)}\right)\right)=(0.4148,0.2696,0.1618,0.1008,0.0530) .
$$

Furthermore, wehave $w_{1}\left(\bar{\alpha}_{\sigma(1)}\right)>w_{1}\left(\bar{\alpha}_{\sigma(2)}\right)>w_{1}\left(\bar{\alpha}_{\sigma(3)}\right)>w_{1}\left(\bar{\alpha}_{\sigma(4)}\right)>w_{1}\left(\bar{\alpha}_{\sigma(5)}\right)$ which reflects the monotonicity and DMs' preference, but the scale-invariance.

2. If we use the PWD method to calculate, then we have

$$
\begin{aligned}
& \left(S\left(\bar{\alpha}_{\sigma(1)}\right), S\left(\bar{\alpha}_{\sigma(2)}\right), S\left(\bar{\alpha}_{\sigma(3)}\right), S\left(\bar{\alpha}_{\sigma(4)}\right), S\left(\bar{\alpha}_{\sigma(5)}\right)\right)=(0.65,0.625,0.60,0.60,0.55) ; \\
& \left(H\left(\bar{\alpha}_{\sigma(1)}\right), H\left(\bar{\alpha}_{\sigma(2)}\right), H\left(\bar{\alpha}_{\sigma(3)}\right), H\left(\bar{\alpha}_{\sigma(4)}\right), H\left(\bar{\alpha}_{\sigma(5)}\right)\right)=(1.00,1.00,0.35,0.30,1.00) ; \\
& \left(L\left(\bar{\alpha}_{\sigma(1)}\right), L\left(\bar{\alpha}_{\sigma(2)}\right), L\left(\bar{\alpha}_{\sigma(3)}\right), L\left(\bar{\alpha}_{\sigma(4)}\right), L\left(\bar{\alpha}_{\sigma(5)}\right)\right)=(1.00,1.00,1.00,1.00,0.30) ; \\
& \left(R\left(\bar{\alpha}_{\sigma(1)}\right), R\left(\bar{\alpha}_{\sigma(2)}\right), R\left(\bar{\alpha}_{\sigma(3)}\right), R\left(\bar{\alpha}_{\sigma(4)}\right), R\left(\bar{\alpha}_{\sigma(5)}\right)\right)=(0.35,0.35,1.00,1.00,1.00) .
\end{aligned}
$$

Therefore,

$$
\begin{aligned}
& \left(P\left(\bar{\alpha}_{\sigma(1)}\right), P\left(\bar{\alpha}_{\sigma(2)}\right), P\left(\bar{\alpha}_{\sigma(3)}\right), P\left(\bar{\alpha}_{\sigma(4)}\right), P\left(\bar{\alpha}_{\sigma(5)}\right)\right)=(0.2275,0.2188,0.2100,0.1800,0.1650) ; \\
& \left(w\left(\bar{\alpha}_{\sigma(1)}\right), w\left(\bar{\alpha}_{\sigma(2)}\right), w\left(\bar{\alpha}_{\sigma(3)}\right), w\left(\bar{\alpha}_{\sigma(4)}\right), w\left(\bar{\alpha}_{\sigma(5)}\right)\right)=(0.2272,0.2185,0.2097,0.1798,0.1648) .
\end{aligned}
$$

Furthermore, we have $w\left(\bar{\alpha}_{\sigma(1)}\right)>w\left(\bar{\alpha}_{\sigma(2)}\right)>w\left(\bar{\alpha}_{\sigma(3)}\right)>w\left(\bar{\alpha}_{\sigma(4)}\right)>w\left(\bar{\alpha}_{\sigma(5)}\right)$ and

$$
\frac{w\left(\bar{\alpha}_{\sigma(1)}\right)}{w\left(\bar{\alpha}_{\sigma(2)}\right)}=\frac{s_{2}\left(\bar{\alpha}_{\sigma(1)}\right)}{s_{2}\left(\bar{\alpha}_{\sigma(2)}\right)}=1.0400, \frac{w\left(\bar{\alpha}_{\sigma(3)}\right)}{w\left(\bar{\alpha}_{\sigma(4)}\right)}=\frac{h_{2}\left(\bar{\alpha}_{\sigma(3)}\right)}{h_{2}\left(\bar{\alpha}_{\sigma(4)}\right)}=1.667 \text {, }
$$

which reflects the monotonicity, DMs' preference and scale-invariance. For the third property, the calculation weight of size and proportion by PWD method is similar to that by IIFN this also is the advantage in comparison to prioritized weighted method and other weighted methods. 


\section{Interval-valued intuitionistic fuzzy ordered precise weighted aggregation operators}

Based on Definition 4, we give the definitions of IIFOPWA and IIFOPWG as follows:

Definition 6. Let $\bar{\alpha}_{i}=\left(\left[a_{i}, b_{i}\right],\left[c_{i}, d_{i}\right]\right) \quad(i=1,2, \cdots, n)$ be a collection of IIFNs, interval-valued intuitionistic fuzzy order precise weighted averaging (IIFOPWA) operator and the interval-valued intuitionistic fuzzy order precise weighted geometric (IIFOPWG) operator are defined as follows:

$$
\begin{gathered}
\operatorname{IIFOPWA}\left(\bar{\alpha}_{1}, \bar{\alpha}_{2}, \cdots, \bar{\alpha}_{n}\right)=w_{\sigma(1)} \bar{\alpha}_{\sigma(1)} \oplus w_{\sigma(2)} \bar{\alpha}_{\sigma(2)} \oplus \cdots \oplus w_{\sigma(n)} \bar{\alpha}_{\sigma(n)} ; \\
\operatorname{IIFOPWG}\left(\bar{\alpha}_{1}, \bar{\alpha}_{2}, \cdots, \bar{\alpha}_{n}\right)=\bar{\alpha}_{\sigma(1)}^{w_{\sigma(1)}} \otimes \bar{\alpha}_{\sigma(2)}^{w_{\sigma(2)}} \otimes \cdots \otimes \bar{\alpha}_{\sigma(n)}^{w_{\sigma(n)}},
\end{gathered}
$$

where $\left(\bar{\alpha}_{\sigma(1)}, \bar{\alpha}_{\sigma(2)}, \cdots, \bar{\alpha}_{\sigma(n)}\right)$ is a permutation of $\left(\bar{\alpha}_{1}, \bar{\alpha}_{2}, \cdots, \bar{\alpha}_{n}\right)$ such that $\bar{\alpha}_{\sigma(i-1)} \geq \bar{\alpha}_{\sigma(i)}$, and $\left(w_{\sigma(1)}, w_{\sigma(2)}, \cdots, w_{\sigma(n)}\right)$ is precise weight vector of $\bar{\alpha}_{i}$.

In the following, we discuss the relationships and properties of two developed interval-valued intuitionistic fuzzy aggregation operators.

Theorem 3. Let $\bar{\alpha}_{i}=\left(\left[a_{i}, b_{i}\right],\left[c_{i}, d_{i}\right]\right) \quad(i=1,2, \cdots, n)$ be a collection of IIFNs, $\left(w_{\sigma(1)}, w_{\sigma(2)}, \cdots, w_{\sigma(n)}\right)$ is precise weight vector of $\bar{\alpha}_{i}$, and $w=\left(w_{1}, w_{2}, \cdots, w_{n}\right)$ is a reduction permutation of $\left(w_{\sigma(1)}, w_{\sigma(2)}, \cdots, w_{\sigma(n)}\right)$, then

$$
\begin{aligned}
& \operatorname{IIFOPWA}\left(\bar{\alpha}_{1}, \bar{\alpha}_{2}, \cdots, \bar{\alpha}_{n}\right)=\operatorname{IIFWA}\left(\bar{\alpha}_{1}, \bar{\alpha}_{2}, \cdots, \bar{\alpha}_{n}\right) ; \\
& \operatorname{IIFOPWG}\left(\bar{\alpha}_{1}, \bar{\alpha}_{2}, \cdots, \bar{\alpha}_{n}\right)=\operatorname{IIFWG}\left(\bar{\alpha}_{1}, \bar{\alpha}_{2}, \cdots, \bar{\alpha}_{n}\right) .
\end{aligned}
$$

Proof. $\left(\bar{\alpha}_{\sigma(1)}, \bar{\alpha}_{\sigma(2)}, \cdots, \bar{\alpha}_{\sigma(n)}\right)$ is a permutation of $\left(\bar{\alpha}_{1}, \bar{\alpha}_{2}, \cdots, \bar{\alpha}_{n}\right)$, based on Theorem 1 , we have $\left(w_{\sigma(1)}, w_{\sigma(2)}, \cdots, w_{\sigma(n)}\right)$ is a permutation of $\left(w_{1}, w_{2}, \cdots, w_{n}\right)$ such that $w_{\sigma(i-1)} \geq w_{\sigma(i)}$ for $i=2,3, \cdots, n$. Thus, $\left(w_{\sigma(1)} \bar{\alpha}_{\sigma(1)}, w_{\sigma(1)} \bar{\alpha}_{\sigma(2)}, \cdots, w_{\sigma(1)} \bar{\alpha}_{\sigma(n)}\right)$ is also a permutation of $\left(w_{1} \bar{\alpha}_{1}, w_{2} \bar{\alpha}_{2}, \cdots, w_{n} \bar{\alpha}_{n}\right)$ such that $w_{\sigma(i-1)} \bar{\alpha}_{\sigma(i-1)} \geq w_{\sigma(i)} \bar{\alpha}_{\sigma(i)}$, then

$w_{\sigma(1)} \bar{\alpha}_{\sigma(1)} \oplus w_{\sigma(2)} \bar{\alpha}_{\sigma(2)} \oplus \cdots \oplus w_{\sigma(n)} \bar{\alpha}_{\sigma(n)}=w_{1} \bar{\alpha}_{1} \oplus w_{2} \bar{\alpha}_{2} \oplus \cdots \oplus w_{n} \bar{\alpha}_{n}$.

Therefore,

$$
\operatorname{IIFOPWA}\left(\bar{\alpha}_{1}, \bar{\alpha}_{2}, \cdots, \bar{\alpha}_{n}\right)=\operatorname{IIFWA}\left(\bar{\alpha}_{1}, \bar{\alpha}_{2}, \cdots, \bar{\alpha}_{n}\right) .
$$

Similarly, we have:

$$
\operatorname{IIFOPWG}\left(\bar{\alpha}_{1}, \bar{\alpha}_{2}, \cdots, \bar{\alpha}_{n}\right)=\operatorname{IIFWG}\left(\bar{\alpha}_{1}, \bar{\alpha}_{2}, \cdots, \bar{\alpha}_{n}\right) .
$$

Thus the proof is completed.

Theorem 4. Let $\bar{\alpha}_{i}=\left(\left[a_{i}, b_{i}\right],\left[c_{i}, d_{i}\right]\right)(i=1,2, \cdots, n)$ be a collection of IIFNs, then IIFOPWA operator aggregated value is also an IIFN, and

$$
\begin{aligned}
& \operatorname{IIFOPWA}\left(\bar{\alpha}_{1}, \bar{\alpha}_{2}, \cdots, \bar{\alpha}_{n}\right)=w_{\sigma(1)} \bar{\alpha}_{\sigma(1)} \oplus w_{\sigma(2)} \bar{\alpha}_{\sigma(2)} \oplus \cdots \oplus w_{\sigma(n)} \bar{\alpha}_{\sigma(n)}= \\
& \left(\left[1-\prod_{i=1}^{n}\left(1-a_{\sigma(i)}\right)^{w_{\sigma(i)}}, 1-\prod_{i=1}^{n}\left(1-b_{\sigma(i)}\right)^{w_{\sigma(i)}}\right],\left[\prod_{i=1}^{n} c_{\sigma(i)}{ }^{w_{\sigma(i)}}, \prod_{i=1}^{n} d_{\sigma(i)}{ }^{w_{\sigma(i)}}\right]\right),
\end{aligned}
$$

where $\left(w_{\sigma(1)}, w_{\sigma(2)}, \cdots, w_{\sigma(n)}\right)$ is precise weight vector of $\bar{\alpha}_{i}$.

Proof. Based on Theorem 3, we can get:

$$
\operatorname{IIFOPWA}\left(\bar{\alpha}_{1}, \bar{\alpha}_{2}, \cdots, \bar{\alpha}_{n}\right)=\operatorname{IIFWA}\left(\bar{\alpha}_{1}, \bar{\alpha}_{2}, \cdots, \bar{\alpha}_{n}\right) \text {. }
$$


Similar to the proof of IIFWA operator (Xu 2007b), we can get Theorem 4 easily.

Theorem 5. (Boundness) Let $\bar{\alpha}_{i}=\left(\left[a_{i}, b_{i}\right],\left[c_{i}, d_{i}\right]\right) \quad(i=1,2, \cdots, n)$ be a collection of IIFNs, and $\left(w_{\sigma(1)}, w_{\sigma(2)}, \cdots, w_{\sigma(n)}\right)$ is precise weight vector of $\bar{\alpha}_{i}$. If

$$
\begin{aligned}
& \bar{\alpha}^{-}=\left(\left[\min _{i}\left\{a_{i}\right\}, \min _{i}\left\{b_{i}\right\}\right],\left[\max _{i}\left\{c_{i}\right\}, \max _{i}\left\{d_{i}\right\}\right]\right) ; \\
& \bar{\alpha}^{+}=\left(\left[\max _{i}\left\{a_{i}\right\}, \max _{i}\left\{b_{i}\right\}\right],\left[\min _{i}\left\{c_{i}\right\}, \min _{i}\left\{d_{i}\right\}\right]\right),
\end{aligned}
$$

then

$$
\bar{\alpha}^{-} \leq \operatorname{IIFOPWA}\left(\bar{\alpha}_{1}, \bar{\alpha}_{2}, \cdots, \bar{\alpha}_{n}\right) \leq \bar{\alpha}^{+} .
$$

Proof. Since $\min _{i}\left\{a_{i}\right\} \leq a_{i} \leq \max _{i}\left\{a_{i}\right\}, \min _{i}\left\{b_{i}\right\} \leq b_{i} \leq \max _{i}\left\{b_{i}\right\}, \min _{i}\left\{c_{i}\right\} \leq c_{i} \leq \max _{i}\left\{c_{i}\right\}$ and $\min _{i}\left\{d_{i}\right\} \leq d_{i} \stackrel{i}{\leq} \max _{i}\left\{d_{i}\right\}$, for all $i$, then ${ }^{i}$

$$
\begin{gathered}
1-\prod_{i=1}^{n}\left(1-a_{\sigma(i)}\right)^{w_{\sigma(i)}} \geq 1-\prod_{i=1}^{n}\left(1-\min _{i}\left\{a_{i}\right\}\right)^{w_{\sigma(i)}}= \\
1-\left(1-\min _{i}\left\{a_{i}\right\}\right)^{\sum_{i=1}^{n} w_{\sigma(i)}}=\min _{i}\left\{a_{i}\right\} \\
\prod_{i=1}^{n} c_{\sigma(i)}{ }^{w_{\sigma(i)}} \geq \prod_{i=1}^{n}\left(\min _{i}\left\{c_{i}\right\}\right)^{w_{\sigma(i)}}=\left(\min _{i}\left\{c_{i}\right\}\right)^{\sum_{i=1}^{n} w_{\sigma(i)}}=\min _{i}\left\{c_{i}\right\} .
\end{gathered}
$$

Similarly, we have:

$$
\begin{gathered}
1-\prod_{i=1}^{n}\left(1-a_{\sigma(i)}\right)^{w_{\sigma(i)}} \leq \max _{i}\left\{a_{i}\right\} ; \\
\prod_{i=1}^{n} c_{\sigma(i)}{ }^{w_{\sigma(i)}} \leq \max _{i}\left\{c_{i}\right\} ; \\
\min _{i}\left\{b_{i}\right\} \leq 1-\prod_{i=1}^{n}\left(1-b_{\sigma(i)}\right)^{w_{\sigma(i)}} \leq \max _{i}\left\{b_{i}\right\} ; \\
\min _{i}\left\{d_{i}\right\} \leq \prod_{i=1}^{n} d_{\sigma(i)}{ }^{w_{\sigma(i)}} \leq \max _{i}\left\{d_{i}\right\} .
\end{gathered}
$$

Set IIFOPWA $\left(\bar{\alpha}_{1}, \bar{\alpha}_{2}, \cdots, \bar{\alpha}_{n}\right)=\bar{\alpha}=([a, b],[c, d])$, then we can get:

$$
\begin{aligned}
& s_{2}(\bar{\alpha}) \leq \frac{2+\max _{i}\left\{a_{i}\right\}-\min _{i}\left\{c_{i}\right\}+\max _{i}\left\{b_{i}\right\}-\min _{i}\left\{d_{i}\right\}}{4}=s_{2}\left(\bar{\alpha}^{+}\right) ; \\
& s_{2}(\bar{\alpha}) \geq \frac{2+\min _{i}\left\{a_{i}\right\}-\max _{i}\left\{c_{i}\right\}+\min _{i}\left\{b_{i}\right\}-\max _{i}\left\{d_{i}\right\}}{4}=s_{2}\left(\bar{\alpha}^{-}\right) ;
\end{aligned}
$$

(1) If $s_{2}(\bar{\alpha})<s_{2}\left(\bar{\alpha}^{+}\right)$and $s_{2}(\bar{\alpha})>s_{2}\left(\bar{\alpha}^{-}\right)$, we have:

$$
\bar{\alpha}^{-}<\operatorname{IIFOPWA}\left(\bar{\alpha}_{1}, \bar{\alpha}_{2}, \cdots, \bar{\alpha}_{n}\right)<\bar{\alpha}^{+} ;
$$

(2) If $s_{2}(\bar{\alpha})=s_{2}\left(\bar{\alpha}^{+}\right)$, we have $a=\max _{i}\left\{a_{i}\right\}, b=\max _{i}\left\{b_{i}\right\}, c=\min _{i}\left\{c_{i}\right\}, d=\min _{i}\left\{d_{i}\right\}$ and

$$
h_{2}(\bar{\alpha})=\frac{a+b+c+d}{4}=h_{2}\left(\bar{\alpha}^{+}\right) \text {. }
$$

Based on the order relation between two IIFNs (Xu, Yager 2006), we have: 


$$
\operatorname{IIFOPWA}\left(\bar{\alpha}_{1}, \bar{\alpha}_{2}, \cdots, \bar{\alpha}_{n}\right) \sim \bar{\alpha}^{+}
$$

(3) If $s_{2}(\bar{\alpha})=s_{2}\left(\bar{\alpha}^{-}\right)$, we have $a=\min _{i}\left\{a_{i}\right\}, b=\min _{i}\left\{b_{i}\right\}, c=\max _{i}\left\{c_{i}\right\}, d=\max _{i}\left\{d_{i}\right\}$ and

$$
h_{2}(\bar{\alpha})=\frac{a+b+c+d}{4}=h_{2}\left(\bar{\alpha}^{-}\right)
$$

$$
\operatorname{IIFOPWA}\left(\bar{\alpha}_{1}, \bar{\alpha}_{2}, \cdots, \bar{\alpha}_{n}\right) \sim \bar{\alpha}^{-} \text {. }
$$

From the above three phases, (1) (2) and (3), we have:

$$
\bar{\alpha}^{-} \leq \operatorname{IIFOPWA}\left(\bar{\alpha}_{1}, \bar{\alpha}_{2}, \cdots, \bar{\alpha}_{n}\right) \leq \bar{\alpha} .
$$

This completes the proof of Theorem 5 .

Theorem 6. (Commutativity) Let $\bar{\alpha}_{i}=\left(\left[a_{i}, b_{i}\right],\left[c_{i}, d_{i}\right]\right)(i=1,2, \cdots, n)$ be a collection of IFFNs, and $\left(\bar{\alpha}_{1}^{\prime}, \bar{\alpha}_{2}^{\prime}, \cdots, \bar{\alpha}_{n}^{\prime}\right)$ is any permutation of $\left(\bar{\alpha}_{1}, \bar{\alpha}_{2}, \cdots, \bar{\alpha}_{n}\right)$, then

$$
\operatorname{IIFOPWA}\left(\bar{\alpha}_{1}^{\prime}, \bar{\alpha}_{2}^{\prime}, \cdots, \bar{\alpha}_{n}^{\prime}\right)=\operatorname{IIFOPWA}\left(\bar{\alpha}_{1}, \bar{\alpha}_{2}, \cdots, \bar{\alpha}_{n}\right) \text {. }
$$

Proof. For $\left(\bar{\alpha}_{1}^{\prime}, \bar{\alpha}_{2}^{\prime}, \cdots, \bar{\alpha}_{n}^{\prime}\right)$ is a permutation of $\left(\bar{\alpha}_{1}, \bar{\alpha}_{2}, \cdots, \bar{\alpha}_{n}\right)$, we have:

$$
\left(\bar{\alpha}_{\sigma(1)}^{\prime}, \bar{\alpha}_{\sigma(2)}^{\prime}, \cdots, \bar{\alpha}_{\sigma(n)}^{\prime}\right)=\left(\bar{\alpha}_{\sigma(1)}, \bar{\alpha}_{\sigma(2)}, \cdots, \bar{\alpha}_{\sigma(n)}\right) \text {, }
$$

where $\left(\bar{\alpha}_{\sigma(1)}^{\prime}, \bar{\alpha}_{\sigma(2)}^{\prime}, \cdots, \bar{\alpha}_{\sigma(n)}^{\prime}\right)$ is a permutation of $\left(\bar{\alpha}_{1}^{\prime}, \bar{\alpha}_{2}^{\prime}, \cdots, \bar{\alpha}_{n}^{\prime}\right)$ with $\bar{\alpha}_{\sigma(i-1)}^{\prime} \geq \bar{\alpha}_{\sigma(i)}^{\prime}$, and $\left(\bar{\alpha}_{\sigma(1)}, \bar{\alpha}_{\sigma(2)}, \cdots, \bar{\alpha}_{\sigma(n)}\right)$ is a permutation of $\left(\bar{\alpha}_{1}, \bar{\alpha}_{2}, \cdots, \bar{\alpha}_{n}\right)$ such that $\alpha_{\sigma(i-1)}>\alpha_{\sigma(i)}$, for $i=2,3, \cdots, n$. By Eqs (6)-(13), we have:

$$
\left(\bar{w}_{\sigma(1)}, \bar{w}_{\sigma(2)}, \cdots, \bar{w}_{\sigma(n)}\right)=\left(w_{\sigma(1)}, w_{\sigma(2)}, \cdots, w_{\sigma(n)}\right) .
$$

Therefore,

$$
\operatorname{IIFOPWA}\left(\bar{\alpha}_{1}^{\prime}, \bar{\alpha}_{2}^{\prime}, \cdots, \bar{\alpha}_{n}^{\prime}\right)=\operatorname{IIFOPWA}\left(\bar{\alpha}_{1}, \bar{\alpha}_{2}, \cdots, \bar{\alpha}_{n}\right) .
$$

This completes the proof of Theorem 6 .

Theorem 7. (Idempotency) Let $\bar{\alpha}_{i}=\left(\left[a_{i}, b_{i}\right],\left[c_{i}, d_{i}\right]\right) \quad(i=1,2, \cdots, n)$ be a collection of IIFNs, and $\left(w_{\sigma(1)}, w_{\sigma(2)}, \cdots, w_{\sigma(n)}\right)$ is precise weight vector of $\bar{\alpha}_{i}$. If all $\bar{\alpha}_{i}(i=1,2, \cdots, n)$ are equal, i.e. $\bar{\alpha}_{i}=\bar{\alpha}=([a, b],[c, d])$, then

$$
\operatorname{IIFOPWA}\left(\bar{\alpha}_{1}, \bar{\alpha}_{2}, \cdots, \bar{\alpha}_{n}\right)=\bar{\alpha}=([a, b],[c, d]) \text {. }
$$

Proof. Since $\bar{\alpha}_{i}=\bar{\alpha}=([a, b],[c, d])(i=1,2, \cdots, n)$, then by Theorem 2, we have:

$$
w_{\sigma(1)}=w_{\sigma(2)}=\cdots=w_{\sigma(n)}=1 / n .
$$

Based on Definition 6, we have:

$$
\operatorname{IIFOPWA}\left(\bar{\alpha}_{1}, \bar{\alpha}_{2}, \cdots, \bar{\alpha}_{n}\right)=w_{\sigma(1)} \bar{\alpha}_{\sigma(1)} \oplus \cdots \oplus w_{\sigma(n)} \bar{\alpha}_{\sigma(n)}=\bar{\alpha},
$$

which complete the proof of the theorem.

Corollary 1. If $\bar{\alpha}_{i}=\left(\left[a_{i}, b_{i}\right],\left[c_{i}, d_{i}\right]\right)(i=1,2, \cdots, n)$ is a collection of the largest IIFNs, i.e. $\bar{\alpha}_{i}=\bar{\alpha}=([1,1],[0,0])$, for all $i$, and $\left(w_{\sigma(1)}, w_{\sigma(2)}, \cdots, w_{\sigma(n)}\right)$ is precise weight vector of $\bar{\alpha}_{i}$, then

$$
\operatorname{IIFOPWA}\left(\bar{\alpha}_{1}, \bar{\alpha}_{2}, \cdots, \bar{\alpha}_{n}\right)=w_{\sigma(1)} \bar{\alpha}_{\sigma(1)} \oplus \cdots \oplus w_{\sigma(n)} \bar{\alpha}_{\sigma(n)}=\bar{\alpha}=([1,1],[0,0]),
$$

which is also the largest IIFN. 
Proof. Similar to the proof of Theorem 7 we can get Corollary easily.

Corollary 2. (Non-compensatory) Assume that $\bar{\alpha}_{i}=\left(\left[a_{i}, b_{i}\right],\left[c_{i}, d_{i}\right]\right)$ is the smallest IIFN, i.e. $\bar{\alpha}_{i}=([0,0],[1,1])$, then

$$
\begin{gathered}
\operatorname{IIFOPWA}\left(\bar{\alpha}_{1}, \bar{\alpha}_{2}, \cdots, \bar{\alpha}_{n}\right)=\operatorname{IIFOPWA}\left(\bar{\alpha}_{2}, \bar{\alpha}_{3}, \cdots, \bar{\alpha}_{n}\right), i=1 ; \\
\operatorname{IIFOPWA}\left(\bar{\alpha}_{1}, \bar{\alpha}_{2}, \cdots, \bar{\alpha}_{n}\right)=\operatorname{IIFOPWA}\left(\bar{\alpha}_{1}, \cdots, \bar{\alpha}_{i-1}, \bar{\alpha}_{i+1}, \cdots, \bar{\alpha}_{n}\right), i=2, \cdots, n .
\end{gathered}
$$

Proof. Since $\bar{\alpha}_{i}=([0,0],[1,1])$, by Definition 5, we have $w\left(\bar{\alpha}_{\sigma(i)}\right) \rightarrow 0^{+}$, then

$$
\begin{aligned}
&\left(1-a_{\sigma(i)}\right)^{w_{\sigma(i)}}=\left(1-b_{\sigma(i)}\right)^{w_{\sigma(i)}}=1 ; \\
& c_{\sigma(i)}{ }^{w_{\sigma(i)}}=d_{\sigma(i)}{ }^{w_{\sigma(i)}}=1 ;
\end{aligned}
$$

$w=\left(w_{\sigma(1)}, \cdots, w_{\sigma(i-1)}, w_{\sigma(i)}, w_{\sigma(i+1)}, \cdots, w_{\sigma(n)}\right)=\left(w_{\sigma(1)}^{\prime}, \cdots, w_{\sigma(i-1)}^{\prime}, 0, w_{\sigma(i+1)}^{\prime}, \cdots, w_{\sigma(n)}^{\prime}\right)$,

where $w=\left(w_{\sigma(1)}, \cdots, w_{\sigma(j-1)}, w_{\sigma(j)}, w_{\sigma(j+1)}, \cdots, w_{\sigma(n)}\right)$ is precise weight vector of $\bar{\alpha}_{i}$, and $\left(w_{\sigma(1)}^{\prime}, \cdots, w_{\sigma(j-1)}^{\prime}, w_{\sigma(j+1)}^{\prime}, \cdots, w_{\sigma(n)}^{\prime}\right)$ is precise weight vector of $\bar{\alpha}_{i}^{\prime}$.

Therefore,

$$
\operatorname{IIFOPWA}\left(\bar{\alpha}_{1}, \bar{\alpha}_{2}, \cdots, \bar{\alpha}_{n}\right)=\operatorname{IIFOPWA}\left(\bar{\alpha}_{1}, \cdots, \bar{\alpha}_{i-1}, \bar{\alpha}_{i+1}, \cdots, \bar{\alpha}_{n}\right) .
$$

The other case $i=1$ can be obtained similarly.

Thus the proof is completed.

Corollary 1 indicates that the alternative or sample could be the best one if every DM thinks so and gives the biggest IIFNs $([1,1],[0,0])$, in group decision making. Corollary 2 shows that the attributes given the smallest IIFN $([0,0],[1,1])$, by evaluation of DMs, will be overlooked.

Based on IIFOPWA and geometric mean, here we define an interval-valued intuitionistic fuzzy precise weighted geometric (IIFOPWG) operator as follows.

Theorem 8. Let $\bar{\alpha}_{i}=\left(\left[a_{i}, b_{i}\right],\left[c_{i}, d_{i}\right]\right) \quad(i=1,2, \cdots, n)$ be a collection of IIFNs, then the IIFOPWG operator aggregated value is also an IIFN, and

$$
\begin{aligned}
& \operatorname{IIFOPWG}\left(\bar{\alpha}_{1}, \bar{\alpha}_{2}, \cdots, \bar{\alpha}_{n}\right)=\bar{\alpha}_{\sigma(1)}^{w_{\sigma(1)}} \otimes \bar{\alpha}_{\sigma(2)}^{w_{\sigma(2)}} \otimes \cdots \otimes \bar{\alpha}_{\sigma(n)}^{w_{\sigma(n)}}= \\
& \left(\left[\prod_{i=1}^{n} a_{\sigma(i)}{ }^{w_{\sigma(i)}}, \prod_{i=1}^{n} b_{\sigma(i)}{ }^{w_{\sigma(i)}}\right],\left[1-\prod_{i=1}^{n}\left(1-c_{\sigma(i)}\right)^{w_{\sigma(i)}}, 1-\prod_{i=1}^{n}\left(1-d_{\sigma(i)}\right)^{w_{\sigma(i)}}\right]\right),
\end{aligned}
$$

where $\left(w_{\sigma(1)}, w_{\sigma(2)}, \cdots, w_{\sigma(n)}\right)$ is precise weight vector.

Proof. The proof of Theorem 8 is similar to Theorem 4 .

Theorem 9. (Boundness) Let $\bar{\alpha}_{i}=\left(\left[a_{i}, b_{i}\right],\left[c_{i}, d_{i}\right]\right) \quad(i=1,2, \cdots, n)$ be a collection of IIFNs, and $\left(w_{\sigma(1)}, w_{\sigma(2)}, \cdots, w_{\sigma(n)}\right)$ is precise weight vector of $\bar{\alpha}_{i}$. If

$$
\begin{aligned}
& \bar{\alpha}^{-}=\left(\left[\min _{i}\left\{a_{i}\right\}, \min _{i}\left\{b_{i}\right\}\right],\left[\max _{i}\left\{c_{i}\right\}, \max _{i}\left\{d_{i}\right\}\right]\right) ; \\
& \bar{\alpha}^{+}=\left(\left[\max _{i}\left\{a_{i}\right\}, \max _{i}\left\{b_{i}\right\}\right],\left[\min _{i}\left\{c_{i}\right\}, \min _{i}\left\{d_{i}\right\}\right]\right),
\end{aligned}
$$

then

$$
\bar{\alpha}^{-} \leq \operatorname{IIFOPWG}\left(\bar{\alpha}_{1}, \bar{\alpha}_{2}, \cdots, \bar{\alpha}_{n}\right) \leq \bar{\alpha}^{+}
$$


Proof. The proof of Theorem 9 is similar to Theorem 5 .

Theorem 10. (Commutativity) Let $\bar{\alpha}_{i}=\left(\left[a_{i}, b_{i}\right],\left[c_{i}, d_{i}\right]\right) \quad(i=1,2, \cdots, n)$ be a collection of IIFS, and $\left(\bar{\alpha}_{1}^{\prime}, \bar{\alpha}_{2}^{\prime}, \cdots, \bar{\alpha}_{n}^{\prime}\right)$ is any permutation of $\left(\bar{\alpha}_{1}, \bar{\alpha}_{2}, \cdots, \bar{\alpha}_{n}\right)$, then

$$
\operatorname{IIFOPWG}\left(\bar{\alpha}_{1}^{\prime}, \bar{\alpha}_{2}^{\prime}, \cdots, \bar{\alpha}_{n}^{\prime}\right)=\operatorname{IIFOPWG}\left(\bar{\alpha}_{1}, \bar{\alpha}_{2}, \cdots, \bar{\alpha}_{n}\right) \text {. }
$$

Proof. The proof of Theorem 10 is similar to Theorem 6 .

Theorem 11. (Idempotency) Let $\bar{\alpha}_{i}=\left(\left[a_{i}, b_{i}\right],\left[c_{i}, d_{i}\right]\right) \quad(i=1,2, \cdots, n)$ be a collection of IIFNs, where $\left(w_{\sigma(1)}, w_{\sigma(2)}, \cdots, w_{\sigma(n)}\right)$ is precise weight vector of $\bar{\alpha}_{i}$. If all $\bar{\alpha}_{i}$ are equal, i.e. $\bar{\alpha}_{i}=\bar{\alpha}=([a, b],[c, d])$, for all $i$, then

$$
\operatorname{IIFOPWG}\left(\bar{\alpha}_{1}, \bar{\alpha}_{2}, \cdots, \bar{\alpha}_{n}\right)=\bar{\alpha}=([a, b],[c, d]) .
$$

Proof. The proof of Theorem 11 is similar to Theorem 7.

Corollary 3. (Non-compensatory) If $\bar{\alpha}_{i}=\left(\left[a_{i}, b_{i}\right],\left[c_{i}, d_{i}\right]\right)=([0,0],[1,1])$ is a smallest IIFN, then

$$
\begin{gathered}
\operatorname{IIFOPWG}\left(\bar{\alpha}_{1}, \bar{\alpha}_{2}, \cdots, \bar{\alpha}_{n}\right)=\operatorname{IIFOPWG}\left(\bar{\alpha}_{2}, \bar{\alpha}_{3}, \cdots, \bar{\alpha}_{n}\right),(i=1) ; \\
\operatorname{IIFOPWG}\left(\bar{\alpha}_{1}, \bar{\alpha}_{2}, \cdots, \bar{\alpha}_{n}\right)=\operatorname{IIFOPWG}\left(\bar{\alpha}_{1}, \cdots, \bar{\alpha}_{i-1}, \bar{\alpha}_{i+1}, \cdots, \bar{\alpha}_{n}\right),(i=2, \cdots, n) .
\end{gathered}
$$

Proof. The proof of Corollary 3 is similar to Corollary 2.

\section{An application of IIFOPWA and IIFOPWG to group decision making}

The 2nd Youth Olympic Games will be held in Nanjing, during August 16-28, 2014. To propagandize the Olympic spirit and show our national culture, the organization committee wants to introduce a chief director of the opening and closing ceremonies. There is a panel with four possible alternatives to choose: (1) $a_{1}$ is the chief director of the Beijing Olympic Games; (2) $a_{2}$ is the chief director of the 16th Asian Olympic Games; (3) $a_{3}$ is a international famous director from the United States; and (4) $a_{4}$ is a famous director from the domestic. The organization committee must make a decision according to five attributes: (1) $c_{1}$ is the morality; (2) $c_{2}$ is the comprehension of Chinese and Nanjing culture; (3) $c_{3}$ is the international popularity and reputation; (4) $c_{4}$ is the work experience; and (5) $c_{5}$ is his master work. This introduction has been raised great attention from the international Olympic committee and Nanjing municipal government and local government official $d_{1}$, organization committee chairman $d_{2}$, master of Chinese culture $d_{3}$, and two professional moviemakers $d_{4}$ and $d_{5}$ sets up the panel of decision makers which will take the whole responsibility for this introduction. The four possible alternatives $a_{i}(i=1,2,3,4)$ are to be evaluated using the interval-valued intuitionistic fuzzy numbers (IIFNs) by five DMs (decision makers) $d_{k}(k=1,2,3,4,5)$. Meanwhile, five attributes $c_{j}(j=1,2,3,4,5)$ are equal, and the weight vector of five attributes is $w=(0.2,0.2,0.2,0.2,0.2)$. However, the weights of five decision makers are completely unknown. Therefore, we could calculate and give a weight vector to five DMs by the precise weight-determined method for the larger score value and accuracy degree means the more information and knowledge about alternatives. Furthermore, we can set or change all the attributes $c_{j}(j=1,2,3,4,5)$ to be the benefit type. Then, we construct the following five interval-valued intuitionistic fuzzy decisions matrices $D_{(k)}=\left(d_{k}^{i j}\right)_{4 \times 5}(k=1,2,3,4,5)$ (Tables $\left.1-5\right)$. 


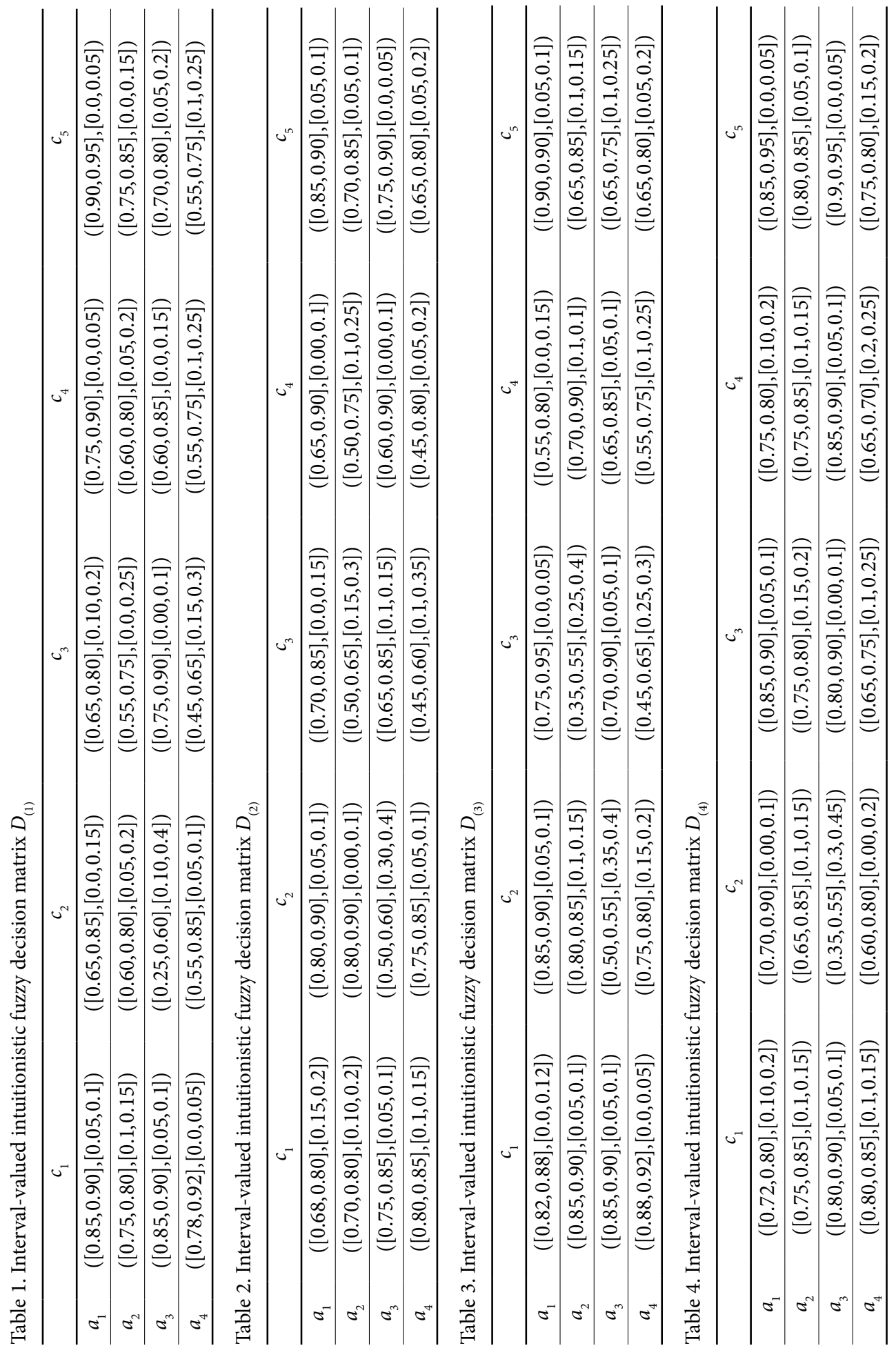


Based on PWD method and IIFOPWA operator, the main steps are as follows:

Step 1: Calculate the score values and accuracy degrees of all IIFNs based on $s_{2}(\bar{\alpha})$ and $h_{2}(\bar{\alpha})$, and construct the interval-valued intuitionistic fuzzy score and accuracy matrices, $S_{(k)}=\left(s_{k}^{i j}\right)_{4 \times 5}$ and $A_{(k)}=\left(\bar{\alpha}_{k}^{i j}\right)_{4 \times 5}(k=1,2,3,4,5)$. Here we only give an interval-valued intuitionistic fuzzy score matrix and accuracy matrix (Tables 6 and 7), and others can be calculated similarly.

Step 2: Calculate precise weight vector

$w\left(d_{\sigma(q)}^{i j}\right)=\left(w\left(d_{\sigma(1)}^{i j}\right), w\left(d_{\sigma(2)}^{i j}\right), w\left(d_{\sigma(3)}^{i j}\right), w\left(d_{\sigma(4)}^{i j}\right), w\left(d_{\sigma(5)}^{i j}\right)\right)$

of $d_{\sigma(q)}^{i j}=\left(d_{\sigma(1)}^{i j}, d_{\sigma(2)}^{i j}, d_{\sigma(3)}^{i j}, d_{\sigma(4)}^{i j}, d_{\sigma(5)}^{i j}\right)$, based on Eqs. (6)-(13), we can get:

$$
\begin{aligned}
& w\left(d_{\sigma(q)}^{11}\right)=(0.2145,0.2133,0.1937,0.1919,0.1865) ; \\
& w\left(d_{\sigma(q)}^{21}\right)=(0.2136,0.2018,0.1988,0.1958,0.1899) ; \\
& w\left(d_{\sigma(q)}^{31}\right)=(0.2040,0.2040,0.2011,0.1955,0.1955) ; \\
& w\left(d_{\sigma(q)}^{41}\right)=(0.2051,0.2022,0.2107,0.1910,0.1910) ; \\
& w\left(d_{\sigma(q)}^{12}\right)=(0.2093,0.2064,0.2035,0.1948,0.1860) ; \\
& w\left(d_{\sigma(q)}^{22}\right)=(0.2130,0.2071,0.2012,0.1923,0.1864) ; \\
& w\left(d_{\sigma(q)}^{32}\right)=(0.2137,0.2051,0.2009,0.1966,0.1838) ; \\
& w\left(d_{\sigma(q)}^{42}\right)=(0.2157,0.2126,0.2032,0.2001,0.1685) ; \\
& w\left(d_{\sigma(q)}^{13}\right)=(0.2211,0.2180,0.2059,0.1843,0.1707) ; \\
& w\left(d_{\sigma(q)}^{23}\right)=(0.2310,0.2202,0.1949,0.1913,0.1625) ; \\
& w\left(d_{\sigma(q)}^{33}\right)=(0.2131,0.2045,0.2017,0.1960,0.1847) ; \\
& w\left(d_{\sigma(q)}^{43}\right)=(0.2210,0.2138,0.1920,0.1884,0.1848) ; \\
& w\left(d_{\sigma(q)}^{14}\right)=(0.2151,0.2093,0.2006,0.1890,0.1860) ; \\
& w\left(d_{\sigma(q)}^{24}\right)=(0.2112,0.2081,0.2050,0.1957,0.1801) ; \\
& w\left(d_{\sigma(q)}^{34}\right)=(0.2211,0.2088,0.2058,0.1835,0.1808) ; \\
& w\left(d_{\sigma(q)}^{44}\right)=(0.2133,0.2000,0.1967,0.1967,0.1933) ; \\
& w\left(d_{\sigma(q)}^{15}\right)=(0.2065,0.2038,0.1984,0.1957,0.1957) ; \\
& w\left(d_{\sigma(q)}^{25}\right)=(0.2047,0.2047,0.2018,0.1988,0.1901) ; \\
& w\left(d_{\sigma(q)}^{35}\right)=(0.2190,0.2104,0.2075,0.1873,0.1758) ; \\
& w\left(d_{\sigma(q)}^{45}\right)=(0.2239,0.2003,0.2003,0.1909,0.1846)
\end{aligned}
$$




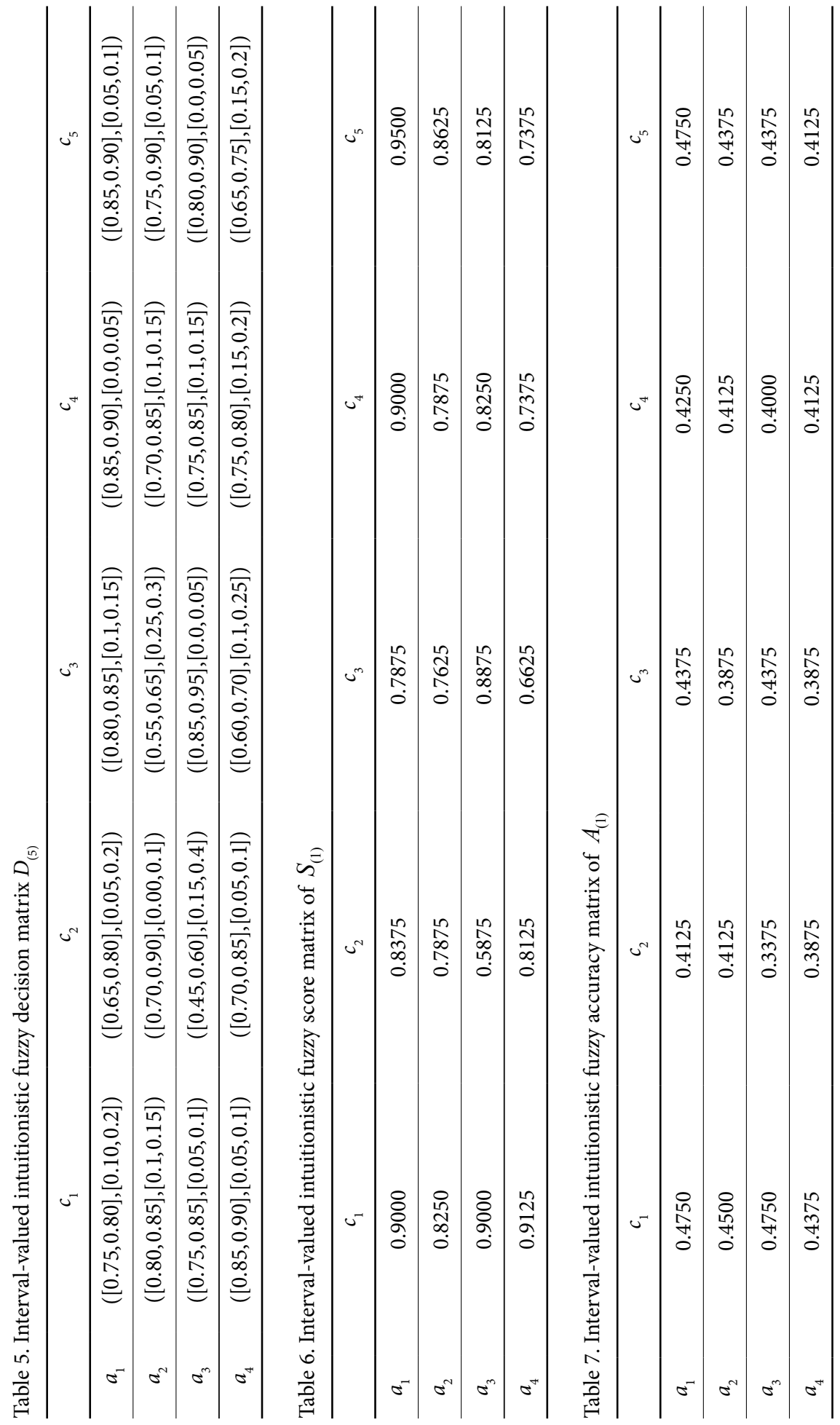


Step 3: Utilize the precise weights $w\left(d_{\sigma(k)}^{i j}\right)$ and IIFOPWA operator to aggregate all interval-valued individual intuitionistic fuzzy decision matrices $D_{(k)}=\left(d_{k}^{i j}\right)_{4 \times 5} \quad(k=1,2,3,4,5)$ into the collective interval-valued intuitionistic fuzzy decision matrix $D=\left(d^{i j}\right)_{4 \times 5}$ (Table 8).

Step 4: Utilize the IIFWA operator to aggregate all preference values $d^{i j} \quad(j=1,2,3,4,5)$ in the $i$ th line of $D$, and get the overall preference values $d^{i} \quad(i=1,2,3,4)$, where $w=(0.20$, $0.20,0.20,0.20,0.20)$.

$$
\begin{aligned}
& d^{1}=([0.7863,0.8837],[0.0000,0.1127]), d^{2}=([0.7019,0.8309],[0.0000,0.1620]), \\
& d^{3}=([0.7205,0.8525],[0.0000,0.1334]), d^{4}=([0.6784,0.8030],[0.0000,0.1759]) .
\end{aligned}
$$

Step 5: Calculate the score $s_{2}\left(d^{i}\right)$ of $d^{i}(i=1,2,3,4)$ respectively, we can get:

$$
s_{2}\left(\bar{\alpha}_{1}\right)=0.8894, s_{2}\left(\bar{\alpha}_{2}\right)=0.8427, s_{2}\left(\bar{\alpha}_{3}\right)=0.8599, s_{2}\left(\bar{\alpha}_{4}\right)=0.8264 \text {. }
$$

Then, $s_{2}\left(\bar{\alpha}_{1}\right)>s_{2}\left(\bar{\alpha}_{3}\right)>s_{2}\left(\bar{\alpha}_{2}\right)>s_{2}\left(\bar{\alpha}_{4}\right)$, and $\bar{\alpha}_{1} \succ \bar{\alpha}_{3} \succ \bar{\alpha}_{2} \succ \bar{\alpha}_{4}$.

Based on PWD method and IIFOPWG operator, the main steps are as follows:

Step $1^{\circ}$ : See Step 1.

Step $2^{\circ}$ : See Step 2.

Step $3^{\circ}$ : Utilize the precise weights $w\left(d_{\sigma(k)}^{i j}\right)$ and IIFOPWG operator to aggregate all the interval-valued individual intuitionistic fuzzy decision matrices $D_{(k)}=\left(d_{k}^{i j}\right)_{4 \times k}(k=1,2,3,4,5)$ into the collective interval-valued intuitionistic fuzzy decision matrix $D=\left(d_{i j}\right)_{4 \times 5}$ (Table 9). Table 8. Collective interval-valued intuitionistic fuzzy decision matrix of $D$

\begin{tabular}{lll}
\hline & $c_{1}$ & $c_{2}$ \\
\hline$a_{1}$ & $([0.7765,0.8439],[0.0000,0.1593])$ & $([0.7486,0.8770],[0.0000,0.1265])$ \\
\hline$a_{2}$ & $([0.7782,0.8463],[0.0877,0.1477])$ & $([0.7241,0.8661],[0.0000,0.1364])$ \\
\hline$a_{3}$ & $([0.8059,0.8828],[0.0500,0.1000])$ & $([0.4193,0.5812],[0.2181,0.4097])$ \\
\hline$a_{4}$ & $([0.8276,0.8936],[0.0000,0.0908])$ & $([0.6825,0.8326],[0.0000,0.1334])$ \\
\hline & $c_{3}$ & $c_{4}$ \\
\hline$a_{1}$ & $([0.7644,0.8853],[0.0000,0.1203])$ & $([0.7333,0.8700],[0.0000,0.0973])$ \\
\hline$a_{2}$ & $([0.5655,0.6956],[0.0000,0.2850])$ & $([0.6636,0.8396],[0.0877,0.1642])$ \\
\hline$a_{3}$ & $([0.7618,0.9060],[0.0000,0.0949])$ & $([0.7103,0.8729],[0.0000,0.1180])$ \\
\hline$a_{4}$ & $([0.5316,0.6759],[0.1318,0.2891])$ & $([0.6076,0.7641],[0.1102,0.2296])$ \\
\hline$a_{1}$ & $([0.8739,0.9257],[0.0000,0.0769])$ & \\
\hline$a_{2}$ & $([0.7367,0.8624],[0.0000,0.1183])$ & \\
\hline$a_{3}$ & $([0.7807,0.8811],[0.0000,0.0921])$ & \\
\hline$a_{4}$ & $([0.6579,0.7822],[0.0908,0.2096])$ & \\
\hline
\end{tabular}


Table 9. Collective interval-valued intuitionistic fuzzy decision matrix of $D$

\begin{tabular}{lll}
\hline & $c_{1}$ & $c_{2}$ \\
\hline$a_{1}$ & $([0.7650,0.8373],[0.0839,0.1671])$ & $([0.7301,0.8708],[0.0314,0.1331])$ \\
\hline$a_{2}$ & $([0.7703,0.8406],[0.0907,0.1518])$ & $([0.7082,0.8605],[0.0525,0.1422])$ \\
\hline$a_{3}$ & $([0.7996,0.8801],[0.0500,0.1000])$ & $([0.3987,0.5799],[0.2478,0.4106])$ \\
\hline$a_{4}$ & $([0.8224,0.8886],[0.0527,0.1028])$ & $([0.6680,0.8305],[0.0629,0.1430])$ \\
\hline & $c_{3}$ & $c_{4}$ \\
\hline$a_{1}$ & $([0.7505,0.8713],[0.0535,0.1339])$ & $([0.7074,0.8608],[0.0224,0.1152])$ \\
\hline$a_{2}$ & $([0.5294,0.6776],[0.1678,0.2958])$ & $([0.6466,0.8303],[0.0907,0.1736])$ \\
\hline$a_{3}$ & $([0.7479,0.8999],[0.0314,0.1010])$ & $([0.6851,0.8701],[0.0414,0.1207])$ \\
\hline$a_{4}$ & $([0.5158,0.6698],[0.1440,0.2924])$ & $([0.5848,0.7603],[0.1233,0.2313])$ \\
\hline$a_{1}$ & $([0.8710,0.9211],[0.0314,0.0814])$ & \\
\hline$a_{2}$ & $([0.7301,0.8604],[0.0517,0.1211])$ & \\
\hline$a_{3}$ & $([0.7567,0.8581],[0.0314,0.1259])$ & \\
\hline$a_{4}$ & $([0.6489,0.7805],[0.1028,0.2108])$ & \\
\hline
\end{tabular}

Step $4^{\circ}$ : Utilize the IIFWG operator to aggregate all preference values $d^{i j}(j=1,2,3,4,5)$ in the $i$-th line of $D$, and get the overall preference values $d^{i}(i=1,2,3,4)$, where $w=(0.20$, $0.20,0.20,0.20,0.20)$.

$$
\begin{aligned}
& d^{1}=([0.7628,0.8718],[0.0448,0.1266]), d^{2}=([0.6713,0.8107],[0.0917,0.1794]), \\
& d^{3}=([0.6583,0.8073],[0.0846,0.1819]), d^{4}=([0.6402,0.7825],[0.0978,0.1988])
\end{aligned}
$$

Step $5^{\circ}$ : Calculate the score $s_{2}\left(d^{i}\right)$ of $d^{i}(i=1,2,3,4)$ respectively, we can get:

$$
s_{2}\left(\bar{\alpha}_{1}\right)=0.8658, s_{2}\left(\bar{\alpha}_{2}\right)=0.8027, s_{2}\left(\bar{\alpha}_{3}\right)=0.7998, s_{2}\left(\bar{\alpha}_{4}\right)=0.7815 \text {. }
$$

Then, $s_{2}\left(\bar{\alpha}_{1}\right)>s_{2}\left(\bar{\alpha}_{2}\right)>s_{2}\left(\bar{\alpha}_{3}\right)>s_{2}\left(\bar{\alpha}_{4}\right)$, and $\bar{\alpha}_{1} \succ \bar{\alpha}_{2} \succ \bar{\alpha}_{3} \succ \bar{\alpha}_{4}$.

Therefore, the optimal chief director of the opening and closing ceremonies is $a_{1}$, i.e. the chief director of the Beijing Olympic Games.

It is pointed out that the ranking of the alternatives have changed, and the sort result by the IIFOPWG operator is different from that by the IIFOPWA operator. The IIFOPWA operator focuses on the impact of overall observed samples and their data, while the IIFOPWG operator highlights the role of individual sample and its data. For example, when we sort by IIFOPWG operator, several smaller attribute values correlated with the alternative $a_{3}$, such as $d_{1}^{32}=([0.25,0.60],[0.10,0.4])$ and $d_{4}^{32}=([0.35,0.55],[0.3,0.45])$ have bigger impact on the variation of its position in the sort. 


\section{Concluding remarks}

In this paper, for putting larger weights into larger IIFNs while keeping the scale-invariant property, we have proposed a precise weight-determined (PWD) method for obtaining IIFN weights based on new score function and accuracy function of IIFN. Since the monotonicity and scale-invariance, the PWD method may be a precise and objective approach to calculate the weights of IIFN and interval-valued intuitionistic fuzzy aggregation operator, and a more suitable approach to distinguish different DMs and experts in group decision making. Based on the PWD method, we have developed two interval-valued intuitionistic fuzzy ordered weighted aggregation operators, i.e. the interval-valued intuitionistic fuzzy ordered precise weighted averaging (IIFOPWA) operator and the interval-valued intuitionistic fuzzy ordered precise weighted geometric (IIFOPWG) operator. We have investigated various properties of PDW method and two new aggregation operators. Finally, an example is given to illustrate the proposed precise weight-determined method, the IIFOWPA operator and the IIFOPWG operator. The work in this paper develops the theories of the interval-valued intuitionistic fuzzy aggregation operators and weight-determined method. It is worth noting that the results of this paper can be extended to hesitant fuzzy environment.

\section{Acknowledgments}

The authors are very grateful to the anonymous reviewers for their insightful and constructive comments and suggestions that have led to an improved version of this paper. This work was supported by National Natural Science Foundation of China (No. 71301141), Humanity and Social Science Youth Foundation of Ministry of Education of China (No. 13YJC630247), Science Application Youth Project of Science and Technology Department of Yunnan Province (No. 2013FD029), and Scientific Research Project of Talent Introduction of Yunnan University of Finance and Economics (No. YC2013D29).

\section{References}

Atanasov, K. T. 1986. Intuitionistic fuzzy sets, Fuzzy Sets and Systems 20(1): 87-96. http://dx.doi.org/10.1016/S0165-0114(86)80034-3

Atanassov, K.; Gargov, G. 1989. Interval-valued intuitionistic fuzzy sets, Fuzzy Sets and Systems 31(3): 343-349. http://dx.doi.org/10.1016/0165-0114(89)90205-4

Cabrerizoa, F. J.; Perezb, I. J.; Herrera, V. E. 2010. Managing the consensus in group decision making in an unbalanced fuzzy linguistic context with incomplete information, Knowledge-Based Systems 23(2): 169-181. http://dx.doi.org/10.1016/j.knosys.2009.11.019

Chen, T. Y.; Hsiao, P. W.; Yen, Y. L. 2011. A multi-criteria group decision-making approach based on interval-valued intuitionistic fuzzy sets: a comparative perspective, Expert Systems with Applications 38(6): 7647-7658. http://dx.doi.org/10.1016/j.eswa.2010.12.096

De, S. K.; Biswas, R.; Roy, A. R. 2000. Some operations on intuitionistic fuzzy sets, Fuzzy Sets and Systems 114: 477-484. http://dx.doi.org/10.1016/S0165-0114(98)00191-2

Deschrijver, G.; Kerre, E. E. 2003. On the relationship between some extensions of fuzzy set theory, Fuzzy Sets and Systems 133(2): 227-235. http://dx.doi.org/10.1016/S0165-0114(02)00127-6 
Ertugrul, I. 2011. Fuzzy group decision making for the selection of facility location, Group Decision and Negotiation 20(6): 725-740. http://dx.doi.org/10.1007/s10726-010-9219-1

Filev, D.; Yager, R. R. 1995. Analytic properties of maximal entropy OWA operators, Information Sciences 85(1-3): 11-27. http://dx.doi.org/10.1016/0020-0255(94)00109-O

Filev, D.; Yager, R. R. 1998. On the issue of obtaining OWA operator weights, Fuzzy Sets and Systems 94(2): 157-169. http://dx.doi.org/10.1016/S0165-0114(96)00254-0

Fuller, R.; Majlender, P. 2003. On obtaining minimal variability OWA operator weights, Fuzzy Sets and Systems 136(2): 203-215. http://dx.doi.org/10.1016/S0165-0114(02)00267-1

Han, Z. S.; Liu, P. 2011. A fuzzy multi-attribute decision-making method under risk with unknown attribute weights, Technological and Economic Development of Economy 17(2): 246-258. http://dx.doi.org/10.3846/20294913.2011.580575

John, P. L.; John, H. K.; Hal, R. A. 2009. Information exchange in group decision making: the hidden profile problem reconsidered, Management Science 55(4): 568-581. http://dx.doi.org/10.1287/mnsc.1080.0975

Kharal, A. 2009. Homeopathic drug selection using intuitionistic fuzzy sets, Homeopathy 98(1): 35-39. http://dx.doi.org/10.1016/j.homp.2008.10.003

Khatibi, V.; Gholam, A. M. 2009. Intuitionistic fuzzy set vs fuzzy set application in medical pattern recognition, Artificial Intelligence in Medicine 47(1): 43-52. http://dx.doi.org/10.1016/j.artmed.2009.03.002

Li, D. F. 2010a. Linear programming method for MADM with interval-valued intuitionistic fuzzy sets, Expert Systems with Applications 37(8): 5939-5945. http://dx.doi.org/10.1016/j.eswa.2010.02.011

Li, D. F. 2010b. TOPSIS-based nonlinear-programming methodology for multi-attribute decision making with interval-valued intuitionistic fuzzy sets, IEEE Transactions on Fuzzy Systems 18(2): 299-311.

Liu, P. D. 2009. Multi-attribute decision-making method research based on interval vague set and TOPSIS method, Technological and Economic Development of Economy 15(3): 453-463. http://dx.doi.org/10.3846/1392-8619.2009.15.453-463

Liu, X.; Chen, L. 2004. On the properties of parametric geometric OWA operator, International Journal of Approximate Reasoning 35(2): 163-178. http://dx.doi.org/10.1016/j.ijar.2003.09.001

Majlender, P. 2005. OWA operators with maximal Renyi entropy, Fuzzy Sets and System 155(3): 340-360. http://dx.doi.org/10.1016/j.fss.2005.04.006

Nettleton, D.; Torra, V. 2001. A comparison of active set method and genetic algorithm approaches for learning weighting vectors in some aggregation operators, International Journal of Intelligent Systems 16(9): 1069-1083. http://dx.doi.org/10.1002/int.1050

O'Hagan, M. 1988. Aggregating template rule antecedents in real time expert systems with fuzzy set logic, in Proc. of the 22nd Annual IEEE Asilomar Conference on Signals, Systems and Computers, Pacific Grove, California, 681-689.

Park, D. G.; Kwun, Y. C.; Park, J. H.; Park, Y. 2009. Correlation coefficient of interval-valued intuitionistic fuzzy sets and its application to multiple attribute group decision making problems, Mathematical and Computer Modelling 50(9-10): 1279-1293. http://dx.doi.org/10.1016/j.mcm.2009.06.010

Vlachos, I. K.; Sergiadis, G. D. 2007. Intuitionistic fuzzy information - Applications to pattern recognition, Pattern Recognition Letters 28(2): 197-206. http://dx.doi.org/10.1016/j.patrec.2006.07.004

Wang, Y. M.; Parkan, C. 2005, A minimax disparity approach for obtaining OWA operator weights, Information Sciences 175(1-2): 20-29. http://dx.doi.org/10.1016/j.ins.2004.09.003

Wang, Y. M.; Parkan, C. 2007. A preemptive goal programming method for aggregating OWA operator weights in group decision making, Information Sciences 177(8): 1867-1877.

http://dx.doi.org/10.1016/j.ins.2006.07.023

Wei, G. W.; Wang, X. R. 2007. Some geometric aggregation operators based on interval-valued intuitionistic fuzzy sets and their application to group decision making, in Proc. of the 2007 international Conference on Computational Intelligence and Security, 495-499. http://dx.doi.org/10.1109/CIS.2007.84 
$\mathrm{Xu}$, Y. J.; Sun, T. 2011. Intuitionistic fuzzy prioritized OWA operator and its application in multi-criteria decision-mading problems, Control and Decision 26(1): 129-132.

$\mathrm{Xu}, \mathrm{Z}$. S. 2007a. Intuitionistic preference relations and their application in group decision making, Information Sciences 177(11): 2363-2379. http://dx.doi.org/10.1016/j.ins.2006.12.019

$\mathrm{Xu}, \mathrm{Z}$. S. 2007b. Intuitionistic fuzzy aggregation operators, IEEE Transactions on Fuzzy Systems 15(6): 1179-1187. http://dx.doi.org/10.1109/TFUZZ.2006.890678

$\mathrm{Xu}, \mathrm{Z}$. S. 2010a. Deviation-based approach to intuitionistic fuzzy multiple attribute group decision making, Group Decision and Negotiation 19(1): 57-76. http://dx.doi.org/10.1007/s10726-009-9164-z

$\mathrm{Xu}, \mathrm{Z}$. S. 2010b. Choquet integrals of weighted intuitionistic fuzzy information, Information Sciences 180(5): 726-736. http://dx.doi.org/10.1016/j.ins.2009.11.011

Xu, Z. S. 2010c. A method based on distance measure for interval-valued intuitionistic fuzzy group decision making, Information Sciences 180(1): 181-190. http://dx.doi.org/10.1016/j.ins.2009.09.005

$\mathrm{Xu}, \mathrm{Z}$. S. 2011. Approaches to multiple attribute group decision making based on intuitionistic fuzzy power aggregation operators, Knowledge-Based Systems 24(6): 749-760. http://dx.doi.org/10.1016/j.knosys.2011.01.011

Xu, Z. S.; Cai, X. Q. 2009. Incomplete interval-valued intuitionistic fuzzy preference relations, International Journal of General Systems 38(8): 871-886. http://dx.doi.org/10.1080/03081070903210630

$\mathrm{Xu}, \mathrm{Z}$. S.; Chen, Q. 2011. A multi-criteria decision making procedure based on interval-valued intuitionistic fuzzy Bonferroni means, Journal of Systems Science and Systems Engineering 20(2): 217-228. http://dx.doi.org/10.1007/s11518-011-5163-0

Xu, Z. S.; Yager, R. R. 2006. Some geometric aggregation operators based on intuitionistic fuzzy sets, International Journal of General Systems 35(4):417-433. http://dx.doi.org/10.1080/03081070600574353

Xu, Z. S.; Chen, J. 2007a. On geometric aggregation over interval-valued intuitionistic fuzzy information, in Proc. of the Fourth International Conference on Fuzzy Systems and Knowledge Discovery, 466-471. http://dx.doi.org/10.1109/FSKD.2007.427

$\mathrm{Xu}, \mathrm{Z}$. S.; Chen, J. 2007b. Approach to group decision making based on interval-valued intuitionistic judgment matrices, Systems Engineering Theory and Practice 27(4): 126-133. http://dx.doi.org/10.1016/S1874-8651(08)60026-5

$\mathrm{Xu}, \mathrm{Z}$. S.; Yager, R. R. 2008. Dynamic intuitionistic fuzzy multi-attribute decision making, International Journal of Approximate Reasoning 48(1): 246-262. http://dx.doi.org/10.1016/j.ijar.2007.08.008

$\mathrm{Xu}, \mathrm{Z}$. S.; Yager, R. R. 2009. Intuitionistic and interval-valued intuitionistic fuzzy preference relations and their measures of similarity for the evaluation of agreement within a group, Fuzzy Optimization and Decision Making 8(2): 123-139. http://dx.doi.org/10.1007/s10700-009-9056-3

Yager, R. R. 1988. On ordered weighted averaging aggregation operators in multi-criteria decision making, IEEE Transactions on Systems Man and Cybernetics 18(1): 183-190. http://dx.doi.org/10.1109/21.87068

Yager, R. R. 2004a. Generalized OWA aggregation operators, Fuzzy Optimization and Decision Making 3: 93-107. http://dx.doi.org/10.1023/B:FODM.0000013074.68765.97

Yager, R. R. 2004b. Modeling prioritized multicriteria decision making, IEEE Transactions on Systems, Man and Cybernetics 34(6): 2396-2404. http://dx.doi.org/10.1109/TSMCB.2004.837348

Ye, J. 2010. Fuzzy decision-making method based on the weighted correlation coefficient under intuitionistic fuzzy environment, European Journal of Operational Research 205(1): 202-204. http://dx.doi.org/10.1016/j.ejor.2010.01.019

Yue, Z. L. 2011. Deriving decision maker's weights based on distance measure for interval-valued intuitionistic fuzzy group decision making, Expert Systems with Applications 38(9): 11665-11670. http://dx.doi.org/10.1016/j.eswa.2011.03.046

Zadeh, L. A. 1965. Fuzzy sets, Information and Control 8(3): 338-353. http://dx.doi.org/10.1016/S0019-9958(65)90241-X 
Zavadskas, E. K.; Turskis, Z. 2011. Multiple criteria decision making (MCDM) methods in economics: an overview, Technological and Economic Development of Economy 17(2): 397-427. http://dx.doi.org/10.3846/20294913.2011.593291

Zhou, W.; He, J. M. 2012. Intuitionistic fuzzy geometric Bonferroni Means and their application in multi-criteria decision making, International Journal of Intelligent Systems 27(12): 995-1019. http://dx.doi.org/10.1002/int.21558

Wei ZHOU received the $\mathrm{PhD}$ degree in management science and engineering from Southeast University, Nanjing, China, in 2012. He is also currently a Vice Professor with the International Business School, Yunnan University of Finance and Economics, Kunming. His research interest covers management engineering and financial engineering.

Jian Min HE received the BSc degree in management science and engineering from Southeast University, Nanjing, China, in 1997. He is also currently a Professor at the School of Economics and Administration, Southeast University. His research interest covers management engineering, systems engineering and financial engineering. 